\title{
Heat and Electricity Market Coordination: A Scalable Complementarity Approach
}

\author{
Mitridati, Lesia Marie-Jeanne Mariane; Kazempour, Jalal; Pinson, Pierre
}

Published in:

European Journal of Operational Research

Link to article, DOI:

10.1016/j.ejor.2019.11.072

Publication date:

2020

Document Version

Peer reviewed version

Link back to DTU Orbit

Citation (APA):

Mitridati, L. M-J. M., Kazempour, J., \& Pinson, P. (2020). Heat and Electricity Market Coordination: A Scalable Complementarity Approach. European Journal of Operational Research, 283(3), 1107-1123.

https://doi.org/10.1016/j.ejor.2019.11.072

\section{General rights}

Copyright and moral rights for the publications made accessible in the public portal are retained by the authors and/or other copyright owners and it is a condition of accessing publications that users recognise and abide by the legal requirements associated with these rights.

- Users may download and print one copy of any publication from the public portal for the purpose of private study or research.

- You may not further distribute the material or use it for any profit-making activity or commercial gain

- You may freely distribute the URL identifying the publication in the public portal 


\title{
Heat and Electricity Market Coordination: A Scalable Complementarity Approach
}

\author{
Lesia Mitridati* \\ Georgia Institute of Technology, H. Milton Stewart School of Industrial $\mathcal{G}$ Systems Engineering, 755 Ferst Dr NW, \\ Atlanta, GA 30318, USA \\ Jalal Kazempour, Pierre Pinson** \\ Technical University of Denmark, Department of Electrical Engineering, Elektrovej 325, DK-2800 Kongens Lyngby, \\ Denmark
}

\begin{abstract}
The large penetration of stochastic and non-dispatchable renewable energy sources increases the need for operational flexibility in power systems. Flexibility can be unlocked by aligning the existing interactions and synergies between heat and power systems. However, in the current sequential order of heat and electricity market clearings, the heat market is myopic to its interactions with the electricity market. This paper designs a heat market, aimed at achieving the optimal coordination of heat and power systems while respecting the current market regulations. The proposed electricity-aware heat market yields a soft coordination between heat and power systems by endogenously modeling their interactions in the day-ahead heat market clearing. The proposed market framework requires to solve a hierarchical optimization problem under uncertainty, which can be computationally challenging in large-scale energy systems with many scenarios. To resolve this potential scalability issue, this paper develops an augmented regularized Benders decomposition algorithm. The performance of the proposed market framework is compared against the fully integrated and sequential market frameworks using an ex-post out-of-sample simulation. This comparison reveals that there is a significant room for improvement in the cost-effective operation of the overall energy system. In particular, the proposed electricity-aware heat market framework provides a trade-off between the sequential and fully integrated market frameworks by significantly reducing the inefficiencies in both heat and electricity systems while respecting the current sequence of clearing heat and electricity markets.
\end{abstract}

Keywords: OR in energy; Integrated energy system; Stochastic programming; Hierarchical optimization; Regularized Benders decomposition

\footnotetext{
${ }^{*}$ Corresponding author. Email address lmitridati3@gatech.edu (L. Mitridati)

** This research was partially supported by the Danish Energy Development Programme (EUDP) through the EnergyLab Nordhavn project (64015-0055). Pierre Pinson was additionally supported by IF through CITIES project (DSF- 1305-00027B), while Jalal Kazempour was also supported by EUDP through the CORE project (64017-0005).
} 


\section{Introduction}

There is a broad consensus in the research community that the stochastic, non-dispatchable, and decentralized nature of renewable energy sources challenges the way power systems and electricity markets have traditionally been operated ( $\mathrm{Chu} \&$ Majumdar, 2012). A broad overview of the challenges related to renewable energy integration is available in Taylor et al. (2016); Morales et al. (2013); Ackermann (2005). Beyond the increasing need for operational flexibility in power systems, the growing interactions between different energy systems, e.g., electricity, natural gas and heat systems, bring us to adopt a more holistic approach to further accommodate renewable energy sources in power systems. In particular, the participation of flexible sources, such as heat pumps (HPs) and combined heat and power plants (CHPs), at the interface between heat and electricity markets provides an opportunity for increasing the flexibility of the overall energy system (Lund, 2005; Lund et al., 2010; Meibom et al., 2013; Wang et al., 2017; Pinson et al., 2017). Traditionally, day-ahead heat and electricity markets have been operated sequentially and independently. Due to the strong technical and economic interactions between heat and electricity systems, the sequential and independent market framework induces inefficiencies in both systems. The main reason for this is that the heat-driven dispatch of CHPs and HPs imposes strong constraints within the electricity market clearing through their minimum power production requirements. In China, this inefficiency has caused a significant increase in wind curtailment (Chen et al., 2015). In Nordic countries, the CHPs cover a large share of the heat demand, and therefore the market power of CHPs in the electricity market has impacted electricity prices (Ummels et al., 2007). In return, electricity prices directly impact the competitiveness and market power of CHPs in the heat market. These interdependencies may have a negative impact on heat production costs and $\mathrm{CO}_{2}$ emissions in the heat system (Virasjoki et al., 2018). Therefore, it is essential to improve the coordination between heat and electricity markets in order to support a cost-effective and flexible operation of the overall energy system, and facilitate the integration of additional renewable energy sources.

The issue of heat and electricity market coordination has mostly been addressed in the literature using co-optimization approaches. The fully integrated heat and electricity market proposed by Chen et al. (2015) allows increasing the wind penetration in power systems by exploiting the operational flexibility of CHPs, combined with electric boilers, HPs, and heat storage tanks. Despite providing an ideal benchmark for the optimal operation of the whole energy system, an integrated day-ahead heat and electricity market as a co-optimization problem goes against the current market regulations, and therefore it is impractical. Mitridati \& Pinson (2016) propose an alternative approach for the coordination of heat and electricity markets based on a bilevel optimization, which exploits the interdependencies between heat and electricity systems while respecting the sequential order of their dispatch. The authors argue that this alternative heat market design facilitates the additional wind power integration by enabling the heat market to anticipate the impact of the heat dispatch of CHPs on the day-ahead electricity market, and in return, the impact of electricity prices on the merit order in the heat market. However, due to the computational complexity of the resulting hierarchical optimization problem, the impact of this market framework on a largescale integrated energy system with high shares of renewable energy penetration is not studied. Similar challenges have been tackled in the literature regarding the coordination of electricity and natural gas systems. Ordoudis et al. (2017) propose a bilevel optimization model for coordination of natural gas and electricity markets, relying on the optimal adjustment of natural gas prices to

improve the scheduling of power plants. Byeon \& Van Hentenryck (2019b) propose a gas-aware unit commitment model using a trilevel optimization, which accounts for the impact of gas prices on 
the profitability of the bids of gas-fired power plants in the electricity market through the so-called bid-validity constraints. These studies suggest the need for a better representation of the technical and economic interdependencies between energy systems in order to facilitate their coordination in a sequential market environment.

Building on the existing literature, the objective of this paper is to develop an adequate market framework for the optimal coordination of heat and electricity systems that can be efficiently implemented in large-scale energy systems. In particular, we answer two main technical questions: First, what is the value of enhancing the coordination between heat and electricity systems in terms of cost-effective operation of the whole energy system with high penetration of renewable energy sources? Second, how to harness this value in a market environment while respecting the current market regulations? Unlike fully integrated approaches that require radical changes in the current market regulations, these research questions acknowledge the necessity of respecting the sequential order of heat and electricity market clearing. Therefore, this study introduces an electricity-aware heat market-clearing model, which endogenously models the interactions between heat and electricity systems in order to reduce inefficiencies in the dispatch of CHPs and HPs. The aim of this approach is to yield a soft coordination between heat and electricity systems by exploiting the interactions and synergies between those systems while respecting the sequential clearing of their respective markets. The proposed market approach provides a trade-off between the current sequential decoupled market framework and a fully integrated one. Comparing the proposed market framework to these two benchmarks provides a basis to quantify the value of increasing the coordination between heat and electricity systems.

The proposed electricity-aware heat market-clearing model requires to solve a hierarchical optimization problem, which is computationally challenging. This model becomes even more challenging to solve if the potential sources of uncertainty in the power system are embedded within this model using a large number of scenarios. Mitridati \& Pinson (2016) take into account uncertainties in the day-ahead electricity market resulting from wind participation, electricity demand and bidding strategy of rival participants. However, the implementation is limited to a small system with a very limited number of scenarios. Since the efficiency of scenario-based stochastic programming relies on the accurate modeling of uncertain parameters by scenarios, this restricts the application of the approach proposed in Mitridati \& Pinson (2016) to systems with a low penetration of renewable energy sources. Therefore, it is essential to provide a computationally tractable reformulation of this electricity-aware heat market clearing in order to study its impact on large-scale integrated energy systems with a high penetration of renewable energy sources.

This motivates us to answer a methodological question: how to enable the proposed electricityaware heat market-clearing model to consider many scenarios and solve it in an efficient and tractable manner? In order to cope with the computational complexity, scenario reduction techniques are convenient to trim down the number of scenarios while retaining the most of the uncertainty description embodied within the original scenario set (Gabriel et al., 2009; Morales et al., 2009). However, scenario reduction might not be sufficient to restore tractability in energy systems with a large number of independent sources of uncertainty. As another alternative, it is possible to exploit the structure of the original optimization problem in order to decompose it into a sequence of simpler and more tractable problems (Castillo et al., 2006). In particular, Benders decomposition has been extensively applied to multi-stage stochastic optimization problems with a large number of scenarios (Benders, 1962; Geoffrion, 1972; Rahmaniani et al., 2017). This class of solution methods has found various applications in power systems. However, it is challenging to generate 
Benders cuts in hierarchical optimization problems. The traditional Benders algorithms generate cuts based on dual variables obtained from the subproblems. However, this approach is not directly applicable to the formulation in Mitridati \& Pinson (2016) with mixed-integer subproblems. Various approaches have been proposed in the literature to tackle such an issue. Wu \& Conejo (2017) propose an alternative method to generate Benders cuts based on the primal variables of the subproblems. However, this method requires to generate a large number of cuts at each iteration, which potentially becomes overwhelming if the algorithm does not converge after a small number of iterations. Alternatively, Kazempour \& Conejo (2012) propose introducing auxiliary subproblems to obtain the optimal value of bilinear terms in a mathematical program with equilibrium constraints including the Karush-Kuhn-Tucker (KKT) conditions. The drawback of this method is that it requires to solve a large number of mixed-integer linear problems at each iteration. In this paper, such an issue is circumvented by reformulating the hierarchical optimization problem using a primal-dual formulation of the lower-level problems instead of the KKT conditions. The bilinear subproblems are then solved sequentially as two Linear Problems (LPs). Additionally, a bundle regularization method introduced by Ruszczynski (1986) and Ruszczynski \& Swietanowski (1997), as well as an augmented master problem formulation proposed by Nasri et al. (2016) are implemented in order to improve the convergence speed of the proposed algorithm. We implement this augmented regularized Benders algorithm in order to study the impact of the electricity-aware heat market framework on an integrated energy system with a large number of scenarios, and discuss its convergence properties.

The remainder of this study is organized as follows. Section 2 discusses the existing interactions and synergies between heat and electricity systems and the challenges to align them in a market environment. Section 3 introduces a novel market framework for the coordination of heat and electricity systems. The computational complexity of the proposed heat market-clearing model, and a scalable solution method based on Benders decomposition are described in Section 4. Section 5 illustrates the impacts of the proposed market-based coordination approach on an integrated energy system for varying values of renewable energy penetration. Finally, Section 6 concludes the study with suggestions for future work. Finally, the notations used throughout the study are listed in an appendix.

\section{Interactions between Heat and Electricity Systems in a Market Environment}

Heat and electricity systems have traditionally been operated independently and sequentially. Despite the decoupled operation of heat and electricity systems, the large penetration of CHPs and HPs at the interface between the systems yields strong economic and physical interactions. This section discusses these existing interactions and the challenges raised for the optimal operation of heat and electricity systems.

\subsection{General Organization of Heat and Electricity Markets}

In Nordic countries such as Denmark, heat and electricity systems are operated sequentially by competitive auction-based markets that interface the technical and economic aspects of each system. These energy markets operate on the principles of power exchanges. Market participants place bids that are dispatched by the market operator based on a merit-order and least-cost principle. Market participants can submit independent hourly price-quantity bids as well as more complex bids, e.g., block orders, that implicitly embed their techno-economic characteristics, such as start-up costs or minimum up and down time. 
The day-ahead heat market is traditionally cleared before the day-ahead electricity market. Heat producers and consumers $j \in \mathcal{I}^{\mathrm{H}}$ submit bids for each hour of the following day $t \in \mathcal{T}$ in form of price-quantity pairs (Varmelast, 2018). Once their day-ahead heat dispatch $Q_{j t}$ is fixed, CHPs and HPs participate in the day-ahead electricity market (Nordpool, 2019). As the day-ahead heat market is cleared prior to the electricity market, day-ahead electricity prices $\lambda_{t \nu}^{\mathrm{E}}$ and dispatch of CHPs $P_{j t \nu}$ and HPs $L_{j t \nu}^{\mathrm{HP}}$ are still uncertain. This uncertainty is represented by the set of scenarios $\nu \in \mathcal{X}$. After the day-ahead electricity market clearing, market participants who need to adjust their heat dispatch participate in the intraday heat markets. These redispatch steps allow market participants to adjust their bids in light of new information on electricity prices and dispatch, and the market operator to update the hourly heat production $Q_{j t \nu}$ based on these updated bids.

Although heat and electricity systems are operated independently, the participation of CHPs and HPs at the interface between both markets induces implicit physical and economic interactions. These interactions are discussed in the following subsections.

\subsection{Physical Interactions}

The physical characteristics of CHPs and HPs induce a strong linkage between heat and electricity production. The majority of CHPs $j \in \mathcal{I}^{\mathrm{CHP}}$ in real world are extraction units that can produce heat $Q_{j t \nu}$ and electricity $P_{j t \nu}$ at varying heat-to-power ratios over each hour of the following day $t \in \mathcal{T}$ under any scenario $\nu \in \mathcal{X}$ of electricity market clearing (Lahdelma \& Hakonen, 2003). Their joint feasible operating region (FOR) is modeled by the minimum heat-to-power ratio $r_{j}$ in (1a), the maximum heat production $\bar{Q}_{j}$ in (1b), and the maximum fuel intake $\bar{F}_{j}$ in (1c), expressed as a linear function of heat and electricity production with parameters $\rho_{j}^{\mathrm{H}}$ and $\rho_{j}^{\mathrm{E}}$, such that

$$
\begin{aligned}
& P_{j t \nu} \geq r_{j} Q_{j t \nu}, \forall j \in \mathcal{I}^{\mathrm{CHP}}, t \in \mathcal{T}, \nu \in \mathcal{X} \\
& 0 \leq Q_{j t \nu} \leq \bar{Q}_{j}, \forall j \in \mathcal{I}^{\mathrm{CHP}}, t \in \mathcal{T}, \nu \in \mathcal{X} \\
& \rho_{j}^{\mathrm{H}} Q_{j t \nu}+\rho_{j}^{\mathrm{E}} P_{j t \nu} \leq \bar{F}_{j}, \forall j \in \mathcal{I}^{\mathrm{CHP}}, t \in \mathcal{T}, \nu \in \mathcal{X} .
\end{aligned}
$$

Additionally, the heat production $Q_{j t \nu}$ of HPs $j \in \mathcal{I}^{\mathrm{HP}}$ is linked to their electricity consumption $L_{j t \nu}^{\mathrm{HP}}$ by their coefficient of performance $\mathrm{COP}_{j}$ in $(2 \mathrm{a})$, and limited by their maximum heat production $\bar{Q}_{j}$ in $(2 \mathrm{~b})$, such that

$$
\begin{aligned}
& Q_{j t \nu}=\mathrm{COP}_{j} L_{j t \nu}^{\mathrm{HP}}, \forall j \in \mathcal{I}^{\mathrm{HP}}, t \in \mathcal{T}, \nu \in \mathcal{X} \\
& 0 \leq Q_{j t \nu} \leq \bar{Q}_{j}, \forall j \in \mathcal{I}^{\mathrm{HP}}, t \in \mathcal{T}, \nu \in \mathcal{X} .
\end{aligned}
$$

As CHPs and HPs interface heat and electricity markets, they can provide flexibility to the integrated energy system. However, in the current sequential and decoupled market framework, the strong linkage between their heat and electricity outputs may limit their flexibility (Chen et al., 2015). In the day-ahead electricity market, extraction CHPs are constrained by their day-ahead heat dispatch $Q_{j t}$, such that their minimum power output $\underline{P}_{j}\left(Q_{j t}\right)$ is defined as

$$
\underline{P}_{j}\left(Q_{j t}\right)=r_{j} Q_{j t}, \forall j \in \mathcal{I}^{\mathrm{CHP}}, t \in \mathcal{T},
$$

and their maximum power output $\bar{P}_{j}\left(Q_{j t}\right)$ is computed as

$$
\bar{P}_{j}\left(Q_{j t}\right)=\frac{\bar{F}_{j}-\rho_{j}^{\mathrm{H}} Q_{j t}}{\rho_{j}^{\mathrm{E}}}, \forall j \in \mathcal{I}^{\mathrm{CHP}}, t \in \mathcal{T} .
$$


Finally, HPs must purchase enough electricity to cover their heat production $Q_{j t}$, such that their minimum electricity consumption $\underline{L}_{j}^{\mathrm{HP}}\left(Q_{j t}\right)$ is given by

$$
\underline{L}_{j}^{\mathrm{HP}}\left(Q_{j t}\right)=\frac{Q_{j t}}{\mathrm{COP}_{j}}, \forall j \in \mathcal{I}^{\mathrm{HP}}, t \in \mathcal{T} .
$$

This heat-driven dispatch strongly impacts the participation of CHPs and HPs in the electricity market. Therefore, when clearing the heat market, it is essential to anticipate the impact of heat dispatch on the electricity market by accurately modeling the participation of CHPs and HPs in the day-ahead electricity market.

\subsection{Economic Interactions}

The production costs of CHPs and HPs are intrinsically linked to their heat and electricity outputs. Since HPs produce heat from electricity purchased in the day-ahead electricity market $L_{j t \nu}^{\mathrm{HP}}$, their heat production cost $\Gamma_{j}^{\mathrm{H}}\left(L_{j t \nu}^{\mathrm{HP}}, \lambda_{t \nu}^{\mathrm{E}}\right)$ can be expressed as a function of their electricity consumption and electricity prices, i.e.,

$$
\Gamma_{j}^{\mathrm{H}}\left(L_{j t \nu}^{\mathrm{HP}}, \lambda_{t \nu}^{\mathrm{E}}\right)=\lambda_{t}^{\mathrm{E}} L_{j t \nu}^{\mathrm{HP}}, \forall j \in \mathcal{I}^{\mathrm{HP}}, t \in \mathcal{T}, \nu \in \mathcal{X} .
$$

Similarly, since CHPs simultaneously produce heat and electricity from fossil fuels, their variable production cost in the heat and electricity day-ahead markets $\Gamma_{j}\left(P_{j t \nu}, Q_{j t}\right)$ can be approximately represented by a linear function of their fuel consumption, such that

$$
\Gamma_{j}\left(P_{j t \nu}, Q_{j t}\right)=\alpha_{j}\left(\rho_{j}^{\mathrm{E}} P_{j t \nu}+\rho_{j}^{\mathrm{H}} Q_{j t}\right), \forall j \in \mathcal{I}^{\mathrm{CHP}}, t \in \mathcal{T}, \nu \in \mathcal{X} .
$$

Due to the strong linkage between their heat and electricity outputs, the cost allocation for heat and electricity production is not straightforward. In the current sequential market framework, CHPs are required to compute their day-ahead heat production cost $\Gamma_{j}^{\mathrm{H}}\left(P_{j t \nu}, Q_{j t}, \lambda_{t}^{\mathrm{E}}\right)$ as total day-ahead production cost minus revenues from sales in the day-ahead electricity market, i.e.,

$$
\Gamma_{j}^{\mathrm{H}}\left(P_{j t \nu}, Q_{j t}, \lambda_{t \nu}^{\mathrm{E}}\right)=\left[\alpha_{j}\left(\rho_{j}^{\mathrm{E}} P_{j t \nu}+\rho_{j}^{\mathrm{H}} Q_{j t}\right)-\lambda_{t \nu}^{\mathrm{E}} P_{j t \nu}\right], \forall j \in \mathcal{I}^{\mathrm{CHP}}, t \in \mathcal{T}, \nu \in \mathcal{X} .
$$

When participating in the heat market, i.e., before the electricity market has been cleared, CHPs and HPs must compute their marginal heat production cost in order to derive price-quantity bids. Therefore, the day-ahead electricity dispatch $P_{j t \nu}$ and prices $\lambda_{t \nu}^{\mathrm{E}}$ are still uncertain. In practice, heat market participants use a deterministic forecast of electricity prices $\hat{\lambda}_{t}^{\mathrm{E}}$ as input to derive their expected marginal heat production cost $\alpha_{j}^{\mathrm{H}}\left(\hat{\lambda}_{t}^{\mathrm{E}}\right)$ from (7)-(8) (Pinson et al., 2017; Mitridati \& Pinson, 2016). For relatively low forecasted electricity prices, the marginal heat production cost of extraction CHPs represents the incremental heat production cost at the minimum power-to-heat ratio $r_{j}$. In contrast, for relatively high forecasted electricity prices, it represents the opportunity loss of producing an extra unit of heat at a ratio $\frac{\rho_{j}^{\mathrm{H}}}{\rho_{j}^{\mathrm{E}}}$, such that

$$
\alpha_{j}^{\mathrm{H}}\left(\hat{\lambda}_{t}^{\mathrm{E}}\right)=\left\{\begin{array}{l}
\alpha_{j}\left(\rho_{j}^{\mathrm{E}} r_{j}+\rho_{j}^{\mathrm{H}}\right)-\hat{\lambda}_{t}^{\mathrm{E}} r_{j}, \text { if } \hat{\lambda}_{t}^{\mathrm{E}} \leq \alpha_{j} \rho_{j}^{\mathrm{E}}, \forall j \in \mathcal{I}^{\mathrm{CHP}}, t \in \mathcal{T} \\
\hat{\lambda}_{t}^{\mathrm{E}} \frac{\rho_{j}^{\mathrm{H}}}{\rho_{j}^{\mathrm{E}}}, \text { if } \hat{\lambda}_{t}^{\mathrm{E}} \geq \alpha_{j} \rho_{j}^{\mathrm{E}}, \forall j \in \mathcal{I}^{\mathrm{CHP}}, t \in \mathcal{T} .
\end{array}\right.
$$


Similarly, since the heat-to-power ratio is considered fixed and given by their COP, the expected heat marginal cost of HPs can be expressed as

$$
\alpha_{j}^{\mathrm{H}}\left(\hat{\lambda}_{t}^{\mathrm{E}}\right)=\frac{\hat{\lambda}_{t}^{\mathrm{E}}}{\mathrm{COP}_{j}}, \forall j \in \mathcal{I}^{\mathrm{HP}}, t \in \mathcal{T} .
$$

The above marginal heat production cost calculation allows CHPs and HPs to implicitly account for interdependencies between heat and electricity markets. However, as the heat market is cleared before the electricity market, CHPs and HPs must anticipate the electricity dispatch and spot prices in order to accurately compute their bids on the heat market. In addition, this approach does not account for the impact of the participation of CHPs and HPs in the electricity market. Virasjoki et al. (2018) highlight the influence of the heat dispatch of CHPs on their market power in the electricity market, and in turn, the impact of electricity prices on the merit order in the heat market. For instance, the power output of CHPs in the electricity market is constrained by their day-ahead heat dispatch. Subsequently, they may offer their minimum electricity production $\underline{P}_{j}\left(Q_{j t}\right)$ at the lowest possible price $\underline{\alpha}$, and offer any additional production up to $\bar{P}_{j}\left(Q_{j t}\right)$ at their true electricity marginal cost $\alpha_{j}^{\mathrm{E}}=\alpha_{j} \rho_{j}^{\mathrm{E}}$. In the context of a high penetration of CHPs, this behavior may shift production from other sources such as renewable energy sources. Similarly, the electricity consumption of HPs is constrained by their day-ahead heat dispatch. Thus, they offer the maximum allowed price $\bar{\alpha}$ for their minimum consumption $\underline{L}_{j}^{\mathrm{HP}}\left(Q_{j t}\right)$.

\section{Market-Based Soft Coordination of Heat and Electricity Systems}

The aforementioned bi-directional interactions between heat and electricity systems offer an opportunity to reveal the full operational flexibility of the overall energy system. However, as discussed in Section 2, traditional sequential and decoupled market frameworks may fail to properly anticipate the impact of CHPs and HPs in the electricity market which may result in inefficiencies in both heat and electricity systems. Therefore, existing market mechanisms need to evolve to better account for the growing interdependencies between the systems. This section introduces an electricity-aware heat market-clearing model. The novelty of this approach is to induce a soft coordination between heat and electricity markets. This is in contrast to the fully-integrated coordination approaches introduced in the literature, which require a simultaneous dispatch of heat and electricity systems (Chen et al., 2015; Li et al., 2016b).

\subsection{Market Framework}

Market-based coordination mechanisms must take into consideration the country-specific regulatory frameworks. Therefore, achieving a market-based soft coordination between heat and electricity systems requires aligning the synergies between the systems towards a social choice, while respecting the current sequential order of their dispatch. In particular, the heat market clearing must explicitly model the bidirectional interactions between heat and electricity systems. Additionally, wholesale markets interface the technical and economic aspects of the operation of heat and electricity systems. In particular, it is essential to consider the operational flexibility of CHPs (Zugno et al., 2016; Chen et al., 2015) and HPs (Bach et al., 2016; Meibom et al., 2007). As the focus of this study is on European markets, which operate on the principles of power exchanges, the proposed market framework must provide institutions and trading mechanisms that implicitly embed these techno-economic characteristics through the participation of different actors in heat 
and electricity markets. In particular, the bid formats of CHPs and HPs in the heat market must reflect the linkage between their heat and electricity outputs and costs.

The proposed market framework builds upon the preliminary work of Mitridati \& Pinson (2016), in which day-ahead heat and electricity market-clearing procedures are modeled as two sequential players trying to minimize their own cost. In a game-theoretic framework, this type of interactions can be represented by a Stackelberg game, in which a leader anticipates the reaction of a follower (von Stackelberg, 1934; Simaan \& Cruz, 1973b,a; Cruz, 1978). As the heat market is cleared before the electricity market, it is modeled as the leader, whose objective is to minimize the heat system cost, while anticipating the impacts of heat-side dispatch on the electricity market. Once the heat market has been cleared, the outcomes of electricity market clearing are still unknown. Therefore, each follower represents a scenario $\nu \in \mathcal{X}$ of electricity market clearing. For given heat dispatch decisions, each follower minimizes the electricity system cost under the underlying scenario $\nu \in \mathcal{X}$. The nature of the sources of uncertainty in the electricity market will be further discussed in Section 3.2 .

In this game-theoretic framework, the proposed electricity-aware heat market clearing can be formulated as a hierarchical (bilevel) optimization problem. In the upper-level optimization problem to be solved by the leader, the heat market clearing minimizes the day-ahead heat production cost and the expected redispatch cost, relied on the bids of the heat market participants. This upper-level optimization problem is subject to optimality conditions of the lower-level optimization problems, which represent the day-ahead electricity market clearing for different realizations of the uncertainty. The electricity outputs of CHPs and HPs in the lower-level optimization problems are constrained by their heat dispatch determined in the upper-level problem, as expressed in (3)-(5). This approach allows the heat market clearing to endogenously model the participation of CHPs and HPs in the day-ahead electricity market. Additionally, the heat market clearing endogenously models the impact of the electricity market clearing on heat productions costs by allowing CHPs and HPs to communicate their price bids as functions of their heat and electricity outputs, and electricity prices, as expressed in (6) and (7). The electricity dispatch and prices are modeled as decision variables of the lower-level problems, and provide feedback to the upper-level problem. Figure 1 schematically illustrates this exchange of information (feedback) between the upper- and lower-level problems.

Once the day-ahead heat market has been cleared, CHPs participate in the day-ahead electricity market (step 2), by computing their minimum and maximum electricity production, as formulated in (3)-(5). After the day-ahead electricity market clearing, the heat and electricity outputs of CHPs and HPs might not be feasible. In view of this updated information, an intraday heat redispatch (step 3) might be performed. Note that the day-ahead heat market clearing anticipates the expected redispatch costs over the scenarios of electricity market clearing considered at the day-ahead stage. This market framework is represented schematically in Figure 1, and detailed in the following subsections.

\subsection{Step 1: Electricity-Aware Heat Market Clearing}

The following presents the mathematical formulation of the proposed electricity-aware heat market clearing (step 1).

\subsubsection{Lower-Level Problems}

For a given day-ahead heat dispatch, each lower-level problem $\mathrm{LL}_{\nu}$ represents the day-ahead electricity market clearing under the underlying scenario $\nu \in \mathcal{X}$. We identify three distinct sources 


\begin{tabular}{|c|c|c|c|c|c|c|c|c|}
\hline \multicolumn{2}{|c|}{$\begin{array}{c}\text { CHPs, HPs, heat-only } \\
\text { units }\end{array}$} & \multicolumn{2}{|c|}{ DA heat dispatch } & \multicolumn{2}{|c|}{$\begin{array}{c}\text { CHPs, HPs, } \\
\text { electricity generators }\end{array}$} & $\frac{\text { DA electricity }}{\text { dispatch }}$ & \multicolumn{2}{|c|}{$\begin{array}{c}\text { CHPs, HPs, heat-only } \\
\text { units }\end{array}$} \\
\hline $\begin{array}{l}\text { Heat price- } \\
\text { quantity bid }\end{array}$ & DA heat & dispatch & \multicolumn{2}{|c|}{$\begin{array}{r}\text { Electricity price- } \\
\text { quantity bid }\end{array}$} & $\begin{array}{l}\text { DA elect } \\
\text { dispatch }\end{array}$ & & $\begin{array}{l}\text { Heat price- } \\
\text { quantity bid }\end{array}$ & $\begin{array}{l}\text { Heat } \\
\text { redispatch }\end{array}$ \\
\hline \multicolumn{3}{|c|}{ Step 1: DA heat market } & & \multirow{2}{*}{\multicolumn{2}{|c|}{$\begin{array}{c}\text { Step 2: } \\
\text { DA electricity } \\
\text { market }\end{array}$}} & & \multicolumn{2}{|c|}{ Step 3: } \\
\hline \multicolumn{2}{|c|}{ Upper-level: } & & & & & & \multicolumn{2}{|c|}{$\begin{array}{l}\text { Intraday heat } \\
\text { redispatch }\end{array}$} \\
\hline
\end{tabular}

DA heat dispatch \begin{tabular}{l|l}
$\hat{i}$ DA electricity \\
dispatch and price
\end{tabular}

Lower-levels:

DA electricity market scenarios

Figure 1: Electricity-aware market framework. Step 1: Day-ahead (DA) electricity-aware heat market (upper-level), constrained by electricity market scenarios (lower-level), step 2: DA electricity market for a given realization of the uncertainty, step 3: Intraday heat redispatch for a given realization of the uncertainty. Dotted lines represent endogenous interactions (feedback) between the upper- and lower-level problems in step 1. Solid lines represent flow of information between market participants and market operators.

of uncertainty related to the day-ahead electricity market clearing, namely renewable power generation, electricity demand, and price bids of rival participants. In practice, one should consider all renewable energy sources and their specific dynamic uncertainties, for which extensive literature exists on how to model and forecast (Pinson, 2013; Morales et al., 2013). In order to simplify the exposition of this study, a single source of renewable energy, i.e., wind energy, is considered. Accordingly, the maximum dispatchable generation $\bar{P}_{j t \nu}$ and price offer $\alpha_{j t \nu}^{\mathrm{E}}$ of wind and conventional producers $j \in \mathcal{I}^{\mathrm{E}}$ at time $t \in \mathcal{T}$, electricity demand $L_{t \nu}^{\mathrm{E}}$, as well as the resulting day-ahead electricity market outcomes, i.e., electricity dispatch $P_{j t \nu}$ and spot price $\lambda_{t \nu}^{\mathrm{E}}$, are indexed by scenarios $\nu \in \mathcal{X}$. However, the day-ahead heat dispatch $Q_{j t}$ of CHPs $j \in \mathcal{I}^{\mathrm{CHP}}$, HPs $j \in \mathcal{I}^{\mathrm{HP}}$ and heat-only units $j \in \mathcal{I}^{\mathrm{HO}}$ are independent of scenarios $\nu \in \mathcal{X}$.

The objective of this optimization problem is to minimize the day-ahead electricity production cost (11a), constrained by hourly electricity balance (11b) and production bounds (11c)-(11f), over the set of primal optimization variables $\Omega_{\nu}^{\mathrm{LL}}=\left\{L_{j t \nu}^{\mathrm{HP}}, P_{j t \nu}, P_{j t \nu}^{0}, P_{j t \nu}^{+}\right\}$, representing the day-ahead electricity production $P_{j t \nu}$ of electricity generators, minimum production $P_{j t \nu}^{0}$ and excess production $P_{j t \nu}^{+}$of CHPs, and electricity consumption $L_{j t \nu}^{\mathrm{HP}}$ of HPs. To be consistent with the European market framework, transmission constraints, ramping, and minimum production constraints are not explicitly accounted for in the heat market clearing. Furthermore, as the vast majority of energy in day-ahead European markets is traded through price-quantity bids, block bids linking different hours of the day are neglected in this study (Nordpool, 2019). For simplicity, it is assumed that wind power producers offer their expected production at a zero marginal cost. In practice, the short-term marginal cost of wind power producers is close to zero (Taylor et al., 2016; Morales 
et al., 2013). The lower-level optimization problem $L L_{\nu}$ is formulated as follows:

$$
\begin{array}{ll}
\min _{\Omega_{\nu}^{\mathrm{L}}} \sum_{j \in \mathcal{I}^{\mathrm{E}}, t \in \mathcal{T}} \tilde{\alpha}_{j t \nu}^{\mathrm{E}} P_{j t \nu}+\sum_{j \in \mathcal{I}^{\mathrm{CHP}}, t \in \mathcal{T}}\left(\underline{\alpha}_{j t \nu}^{0}+\alpha_{j}^{\mathrm{E}} P_{j t \nu}^{+}\right)-\bar{\alpha} L_{j t \nu}^{\mathrm{HP}} \\
\text { s.t. } & L_{t \nu}^{\mathrm{E}}+\sum_{j \in \mathcal{I}^{\mathrm{HP}}} L_{j t \nu}^{\mathrm{HP}}=\sum_{j \in \mathcal{I}^{\mathrm{E}}} P_{j t \nu}+\sum_{j \in \mathcal{I}^{\mathrm{CHP}}}\left(P_{j t \nu}^{+}+P_{j t \nu}^{0}\right): \lambda_{t \nu}^{\mathrm{E}}, \forall t \in \mathcal{T} \\
& 0 \leq P_{j t \nu}^{0} \leq \underline{P}_{j}\left(Q_{j t}\right): \underline{\mu}_{j t \nu}, \bar{\mu}_{j t \nu}, \forall j \in \mathcal{I}^{\mathrm{CHP}}, t \in \mathcal{T} \\
& 0 \leq P_{j t \nu}^{+} \leq \bar{P}_{j}\left(Q_{j t}\right)-\underline{P}_{j}\left(Q_{j t}\right): \underline{\mu}_{j t \nu}^{0}, \bar{\mu}_{j t \nu}^{0}, \forall j \in \mathcal{I}^{\mathrm{CHP}}, t \in \mathcal{T} \\
& 0 \leq P_{j t \nu} \leq \bar{P}_{j t \nu}: \underline{\mu}_{j t \nu}, \bar{\mu}_{j t \nu}, \forall j \in \mathcal{I}^{\mathrm{E}}, t \in \mathcal{T} \\
& 0 \leq L_{j t \nu}^{\mathrm{HP}} \leq \underline{L}_{j}^{\mathrm{HP}}\left(Q_{j t}\right): \underline{\mu}_{j t \nu}, \bar{\mu}_{j t \nu}, \forall j \in \mathcal{I}^{\mathrm{HP}}, t \in \mathcal{T} .
\end{array}
$$

Since CHPs are constrained by their day-ahead heat dispatch, they offer their minimum production up to $\underline{P}_{j}\left(Q_{j t}\right)$ at the lowest price $\underline{\alpha}=-€ 500 / \mathrm{MWh}$, and their excess production up to $\bar{P}_{j}\left(Q_{j t}\right)$ at their true electricity marginal $\operatorname{cost} \alpha_{j} \rho_{j}^{\mathrm{E}}$. Their total electricity production is defined as $P_{j t \nu}=P_{j t \nu}^{0}+P_{j t \nu}^{+}$. Similarly, HPs offer the maximum price $\bar{\alpha}=€ 3000 / \mathrm{MWh}$ in the day-ahead electricity market for their minimum power consumption $\underline{L}_{j}^{\mathrm{HP}}\left(Q_{j t}\right)$. The values of the minimum and maximum price bids in this study are derived from the specifications in the Nordpool dayahead electricity market (Nordpool, 2019). The minimum and maximum power production and consumption in (11d)-(11f) are computed as detailed in (3)-(5). Although the day-ahead heat dispatch $Q_{j t}$ is an upper-level decision variable, it is considered in the lower-level problems as a fixed parameter. The dual variables of the lower-level optimization problem are denoted in front of their corresponding constraints. In particular, day-ahead electricity prices $\lambda_{t \nu}^{\mathrm{E}}$ are defined as dual variables of electricity balance constraints (11b). The set of dual optimization variables is $\Xi_{\nu}^{\mathrm{LL}}=\left\{\lambda_{t \nu}^{\mathrm{E}}, \underline{\mu}_{j t \nu}, \bar{\mu}_{j t \nu}, \underline{\mu}_{j t \nu}^{0}, \bar{\mu}_{j t \nu}^{0}\right\}$.

\subsubsection{Upper-Level Problem}

In the upper-level problem, the heat market clearing seeks to minimize the expected day-ahead heat production cost and redispatch cost (12a) over the set of optimization variables $\Omega^{\mathrm{UL}}=$ $\Omega^{\mathrm{DA}} \bigcup_{\nu \in \mathcal{X}} \Omega_{\nu}^{\mathrm{R}} \bigcup_{\nu \in \mathcal{X}} \Omega_{\nu}^{\mathrm{LL}} \bigcup_{\nu \in \mathcal{X}} \Xi_{\nu}^{\mathrm{LL}}$. The set of day-ahead variables $\Omega^{\mathrm{DA}}=\left\{Q_{j t}, S_{j t}, Q_{j t}^{+}, Q_{j t}^{-}\right\}$ includes the heat dispatch $Q_{j t}$ variables of CHPs, HPs, and heat-only units, the state of charge $S_{j t}$, charge $Q_{j t}^{-}$, and discharge $Q_{j t}^{+}$variables of centrally-operated heat storage tanks. The set of heat redispatch variables is defined as

$$
\Omega_{\nu}^{\mathrm{R}}=\left\{Q_{j t \nu}, Q_{j t \nu}^{\uparrow}, Q_{j t \nu}^{\downarrow}, S_{j t \nu}, Q_{j t \nu}^{+}, Q_{j t \nu}^{-}\right\} .
$$

Variables $Q_{j t \nu}^{\uparrow}$ and $Q_{j t \nu}^{\downarrow}$ represent the upward and downward production adjustment of the CHPs, HPs and heat-only units, associated with the redispatch $\operatorname{costs} \alpha_{j t \nu}^{\uparrow}$ and $\alpha_{j t \nu}^{\downarrow}$. Their heat production after redispatch is denoted as $Q_{j t \nu}=Q_{j t}+Q_{j t \nu}^{\uparrow}-Q_{j t \nu}^{\downarrow}$, as defined in (12i). Variables $S_{j t \nu}, Q_{j t \nu}^{+}$, $Q_{j t \nu}^{-}$represent the state of charge, discharge, and charge variables of the centrally-operated heat storage tanks after redispatch. Therefore, the upper-level optimization problem, representing the electricity-aware heat market-clearing problem, is constrained by heat operating constraints at the day-ahead stage (12b)-(12g), redispatch stage (12h)-(12t), and the lower-level problems $(12 \mathrm{u})$. The 
electricity-aware heat market-clearing model can be formulated as follows:

$$
\begin{aligned}
& \min _{\Omega^{\mathrm{UL}}} \sum_{\nu \in \mathcal{X}, t \in \mathcal{T}} \pi_{\nu}\left[\sum_{j \in \mathcal{I}^{\mathrm{HO}}} \alpha_{j} Q_{j t}+\sum_{j \in \mathcal{I}^{\mathrm{CHP}}}\left(\alpha_{j}\left(\rho_{j}^{\mathrm{H}} Q_{j t}+\rho_{j}^{\mathrm{E}} P_{j t \nu}\right)-\lambda_{t \nu}^{\mathrm{E}} P_{j t \nu}\right)\right. \\
& \left.+\sum_{j \in \mathcal{I}^{\mathrm{CHP}} \cup \mathcal{I}^{\mathrm{HO}}}\left(\alpha_{j t \nu}^{\uparrow} Q_{j t \nu}^{\uparrow}-\alpha_{j t \nu}^{\downarrow} Q_{j t \nu}^{\downarrow}\right)+\sum_{j \in \mathcal{I}^{\mathrm{HP}}} \lambda_{t \nu}^{\mathrm{E}} L_{j t \nu}^{\mathrm{HP}}\right] \\
& \text { s.t. } \quad L_{t}^{\mathrm{H}}=\sum_{j \in \mathcal{I}^{\mathrm{HP}} \cup \mathcal{I}^{\mathrm{CHP}} \cup \mathcal{I}^{\mathrm{HO}}} Q_{j t}+\sum_{j \in \mathcal{I}^{\mathrm{HS}}}\left(Q_{j t}^{+}-Q_{j t}^{-}\right), \forall t \in \mathcal{T} \\
& 0 \leq Q_{j t} \leq \bar{Q}_{j}, \forall j \in \mathcal{I}^{\mathrm{HO}} \cup \mathcal{I}^{\mathrm{HP}} \cup \mathcal{I}^{\mathrm{CHP}}, t \in \mathcal{T} \\
& S_{j t}=S_{j(t-1)}+\rho_{j}^{-} Q_{j t}^{-}-\rho_{j}^{+} Q_{j t}^{+}-l_{j}, \forall j \in \mathcal{I}^{\mathrm{HS}}, t \in \mathcal{T} \\
& 0 \leq Q_{j t}^{-} \leq \bar{Q}_{j}, 0 \leq Q_{j t}^{+} \leq \bar{Q}_{j}, \forall j \in \mathcal{I}^{\mathrm{HS}}, t \in \mathcal{T} \\
& \underline{S}_{j} \leq S_{j t} \leq \bar{S}_{j}, \forall j \in \mathcal{I}^{\mathrm{HS}}, t \in \mathcal{T} \\
& S_{j(t=|\mathcal{T}|)} \geq S_{j}^{\text {init }}, \forall j \in \mathcal{I}^{\mathrm{HS}} \\
& L_{t}^{\mathrm{H}}=\sum_{j \in \mathcal{I}^{\mathrm{HO}} \cup \mathcal{I}^{\mathrm{HP}} \cup \mathcal{I}^{\mathrm{CHP}}} Q_{j t \nu}+\sum_{j \in \mathcal{I}^{\mathrm{HS}}}\left(Q_{j t \nu}^{+}-Q_{j t \nu}^{-}\right), \forall t \in \mathcal{T}, \nu \in \mathcal{X} \\
& Q_{j t \nu}=Q_{j t}+Q_{j t \nu}^{\uparrow}-Q_{j t \nu}^{\downarrow}, \forall j \in \mathcal{I}^{\mathrm{HO}} \cup \mathcal{I}^{\mathrm{HP}} \cup \mathcal{I}^{\mathrm{CHP}}, t \in \mathcal{T}, \nu \in \mathcal{X} \\
& 0 \leq Q_{j t \nu}^{\uparrow}, 0 \leq Q_{j t \nu}^{\downarrow}, \forall j \in \mathcal{I}^{\mathrm{HO}} \cup \mathcal{I}^{\mathrm{HP}} \cup \mathcal{I}^{\mathrm{CHP}}, t \in \mathcal{T}, \nu \in \mathcal{X} \\
& 0 \leq Q_{j t \nu} \leq \bar{Q}_{j} \quad \forall j \in \mathcal{I}^{\mathrm{HO}}, t \in \mathcal{T}, \nu \in \mathcal{X} \\
& P_{j t \nu} \geq r_{j} Q_{j t}, \forall j \in \mathcal{I}^{\mathrm{CHP}}, t \in \mathcal{T}, \nu \in \mathcal{X} \\
& \rho_{j}^{\mathrm{H}} Q_{j t}+\rho_{j}^{\mathrm{E}} P_{j t \nu} \leq \bar{F}_{j}, \forall j \in \mathcal{I}^{\mathrm{CHP}}, t \in \mathcal{T}, \nu \in \mathcal{X} \\
& 0 \leq Q_{j t \nu} \leq \bar{Q}_{j}, \forall j \in \mathcal{I}^{\mathrm{CHP}}, t \in \mathcal{T}, \nu \in \mathcal{X} \\
& Q_{j t \nu}=\mathrm{COP}_{j} L_{j t \nu}^{\mathrm{HP}}, \forall j \in \mathcal{I}^{\mathrm{HP}}, t \in \mathcal{T}, \nu \in \mathcal{X} \\
& 0 \leq Q_{j t \nu} \leq \bar{Q}_{j}, \forall j \in \mathcal{I}^{\mathrm{HP}}, t \in \mathcal{T}, \nu \in \mathcal{X} \\
& S_{j t \nu}=S_{j(t-1) \nu}+\rho_{j}^{-} Q_{j t \nu}^{-}-\rho_{j}^{+} Q_{j t \nu}^{+}-l_{j}, \forall j \in \mathcal{I}^{\mathrm{HS}}, t \in \mathcal{T}, \nu \in \mathcal{X} \\
& \underline{S}_{j} \leq S_{j t \nu} \leq \bar{S}_{j}, \forall j \in \mathcal{I}^{\mathrm{HS}}, t \in \mathcal{T}, \nu \in \mathcal{X} \\
& 0 \leq Q_{j t \nu}^{-} \leq \bar{Q}_{j t}, 0 \leq Q_{j t \nu}^{+} \leq \bar{Q}_{j t}, \forall j \in \mathcal{I}^{\mathrm{HS}}, t \in \mathcal{T}, \nu \in \mathcal{X} \\
& S_{j(j(t=|\mathcal{T}|) \nu} \geq S_{j}^{\text {init }}, \forall j \in \mathcal{I}^{\mathrm{HS}}, \nu \in \mathcal{X} \\
& \left\{P_{j t \nu}, L_{j t \nu}^{\mathrm{HP}}, \lambda_{t \nu}^{\mathrm{E}}\right\} \in \arg \min \left\{\mathrm{LL}_{\nu}\right\}, \forall \nu \in \mathcal{X} .
\end{aligned}
$$

At the day-ahead stage, (12b) enforces hourly heat balance, (12c) represents the heat production bounds of all producers, and $(12 \mathrm{~d})-(12 \mathrm{~g})$ impose the operating constraints of heat storage tanks. The centrally-operated heat storage units can be heat producers $\left(Q_{j t}^{+}>0\right)$ when discharging, or consumers $\left(Q_{j t}^{-}>0\right)$ when charging. Although (12d) allows simultaneous charging and discharging, this type of operation is not optimal when prices are positive due to the charging $\left(0<\rho_{j}^{-}<1\right)$ and discharging $\left(\rho_{j}^{+}>1\right)$ loss coefficients. Additionally, a constraint on the charging state of the storage in the last time period is imposed in $(12 \mathrm{~g})$, so that it is not discharged completely over the optimization period. Alternative approaches could be used for the final state of storage problem, in the form of hard and soft constraints, or modification of the objective function. Furthermore, (12h)- 
(12t) ensure that the dispatch of heat producers is feasible after the day-ahead electricity market clearing. Constraint (12h) enforces hourly heat balance, (12i)-(12k) represent heat production bounds, (12l)-(12p) correspond to the FOR of CHPs and HPs, and (12q)-(12t) model heat storage tanks' operating constraints for each scenario of electricity market clearing. For simplicity, in the rest of this study the following redispatch costs for CHPs and heat-only units are considered:

$$
\left\{\begin{array}{l}
\alpha_{j t \nu}^{\uparrow}=1.1 \alpha_{j} \rho_{j}^{\mathrm{H}}, \forall j \in \mathcal{I}^{\mathrm{CHP}} \cup \mathcal{I}^{\mathrm{HO}}, t \in \mathcal{T}, \nu \in \mathcal{X} \\
\alpha_{j t \nu}^{\downarrow}=0.9 \alpha_{j} \rho_{j}^{\mathrm{H}}, \forall j \in \mathcal{I}^{\mathrm{CHP}} \cup \mathcal{I}^{\mathrm{HO}}, t \in \mathcal{T}, \nu \in \mathcal{X},
\end{array}\right.
$$

and for HPs:

$$
\alpha_{j t \nu}^{\uparrow}=\alpha_{j t \nu}^{\downarrow}=\frac{\lambda_{j t \nu}^{\mathrm{E}}}{\mathrm{COP}_{j}}, \forall j \in \mathcal{I}^{\mathrm{HP}}, t \in \mathcal{T}, \nu \in \mathcal{X} .
$$

Equations (12b)-(12t) represent the upper-level constraints, and (12u) represents the lower-level problems, one per scenario. A common approach to recast this hierarchical optimization problem as a single-level optimization problem is to replace the linear lower-level optimization problems by their sufficient and necessary KKT conditions. The complementarity conditions can be linearized by introducing auxiliary binary variables. In addition, the bilinear terms $\lambda_{t \nu}^{\mathrm{E}} L_{j t \nu}^{\mathrm{HP}}$ and $\lambda_{t \nu}^{\mathrm{E}} P_{j t \nu}$ in the objective function of the upper-level problem can be reformulated as a linear expression by using the strong duality theorem (Gabriel et al., 2012). As a result, this hierarchical optimization problem can be eventually solved as a mixed-integer linear program (MILP). The KKT conditions and the equivalent MILP formulation of (12) are provided in an online appendix (Mitridati et al., 2019).

\subsection{Step 2: Day-Ahead Electricity Market Clearing}

Once the heat system has been dispatched, CHPs and HPs participate in the day-ahead electricity market. For a given realization of the uncertainty $\nu \in \mathcal{X}$, the mathematical formulation of the day-ahead electricity market clearing is identical to the lower-level problem $L L_{\nu}$ in (11).

\subsection{Step 3: Intraday Heat Redispatch}

For a given realization of the uncertainty $\nu \in \mathcal{X}$, CHPs and HPs might not be dispatched at their minimum level $\underline{P}_{j}\left(Q_{j t}\right)$ and $\underline{L}_{j}^{\mathrm{HP}}\left(Q_{j t}\right)$ in the electricity market. As a result, their day-ahead heat $\left(Q_{j t}\right)$ and electricity $\left(P_{j t \nu}, L_{j t \nu}^{\mathrm{HP}}\right)$ outputs might not be in their joint FOR, as expressed in (1)-(2). In this case, the heat market operator performs a redispatch of heat sources. The objective of this optimization problem is to minimize the heat redispatch cost (15a), subject to hourly heat balance, heat production bounds, and operating constraint of CHPs, heat-only units, and heat storage tanks (15b). For a given realization of the uncertainty $\nu \in \mathcal{X}$, the heat redispatch problem is formulated as follows:

$$
\begin{array}{ll}
\min _{\Omega_{\nu}^{\mathrm{R}}} & \sum_{j \in \mathcal{I}^{\mathrm{HO} \cup \mathcal{I}^{\mathrm{HP}} \cup \mathcal{I}^{\mathrm{CHP}}}}\left(\alpha_{j t \nu}^{\uparrow} Q_{j t \nu}^{\uparrow}-\alpha_{j t \nu}^{\downarrow} Q_{j t \nu}^{\downarrow}\right) \\
\text { s.t. } & (12 \mathrm{~h})-(12 \mathrm{t}) .
\end{array}
$$




\section{Scalability Strategy: A Benders Decomposition Approach}

As previously discussed, a straightforward approach to solve the electricity-aware heat marketclearing problem is to recast it as a single-level MILP problem. However, the number of auxiliary binary variables used to linearize the complementarity conditions increases proportionally to the number of scenarios considered, i.e., as $2|\mathcal{X}||\mathcal{T}|\left|\mathcal{I}^{\mathrm{CHP}} \cup \mathcal{I}^{\mathrm{HP}} \cup \mathcal{I}^{\mathrm{E}}\right|$. In order to cope with this computational complexity, it is possible to exploit the structure of the original stochastic hierarchical optimization problem (12) in order to decompose it into a set of simpler and more tractable problems. By using a Benders decomposition algorithm, the original problem can be decomposed into $|\mathcal{X}|$ subproblems, one per scenario $\nu \in \mathcal{X}$, by temporarily fixing the so-called complicating variables, i.e., the day-ahead heat dispatch variables $\Omega^{\mathrm{DA}}$. This section discusses the challenges for applying traditional Benders decomposition algorithms to this class of problems, and introduces an augmented regularized Benders algorithm.

\subsection{Structure of the Proposed Benders Decomposition Algorithm}

Benders decomposition is an iterative process. At each iteration, the complicating variables are updated in the so-called master problem (step A), which only includes the constraints related to the day-ahead heat dispatch (12b)-(12f). The value of the objective function of the master problem provides a lower bound for the objective value of the original problem (12). The subproblems, one per scenario, are then solved independently with the fixed value of each complicating variable (step B). The value of objective function of the subproblems plus the dispatch cost obtained in the master problem provides an upper bound for the value of objective function of the original problem (12). The master problem and the subproblems exchange information until the upper and lower bounds converge (step C). In traditional Benders algorithms, the sensitivities of the subproblems with respect to the complicating variables are derived to generate optimality cuts that further constrain the master problem in the following iterations. These optimality cuts represent a linear under-estimator of the subproblems' objective function at each iteration.

As subproblems are still hierarchical optimization problems, this study develops an augmented regularized Benders algorithm to derive the sensitivities. The specificity of the proposed Benders decomposition algorithm is to reformulate the hierarchical subproblems using a primal-dual formulation of the lower-level optimization problems instead of a traditional MILP formulation.

Instability due to excessive oscillations of the upper and lower bounds is a major drawback of Benders decomposition algorithms (Rahmaniani et al., 2017). In the case of non-convex optimization, this may lead to a slow convergence or even convergence to a suboptimal solution. The so-called bundle method introduced by Ruszczynski (1986) and extended by Ruszczynski \& Swietanowski (1997) mitigates the oscillations of the upper bound. This approach adds a quadratic regularization term to the objective function of the master problem to ensure that the solutions remain close to the current reference point. This reference point and the upper bound are updated at a given iteration if the decrease in the expected objective value of the subproblems is deemed significant (step C). This approach potentially reduces the number of iterations for the Benders decomposition algorithm to converge. Although this increases the computational complexity of the master problem, given that the main computational burden resides in solving the large number of subproblems at each iteration, empirical results show that the overall computational cost is reduced (Ruszczynski \& Swietanowski, 1997).

Finally, as suggested by Nasri et al. (2016), at each iteration a new set of auxiliary cuts is introduced in the master problem, creating an additional feedback from the subproblems. This 
new set of constraints only depends on the value of the (primal) variables of the subproblems in the previous iteration. Figure 2 schematically illustrates the structure of the proposed Benders decomposition algorithm. The formulation of each step is provided in the online appendix (Mitridati et al., 2019).

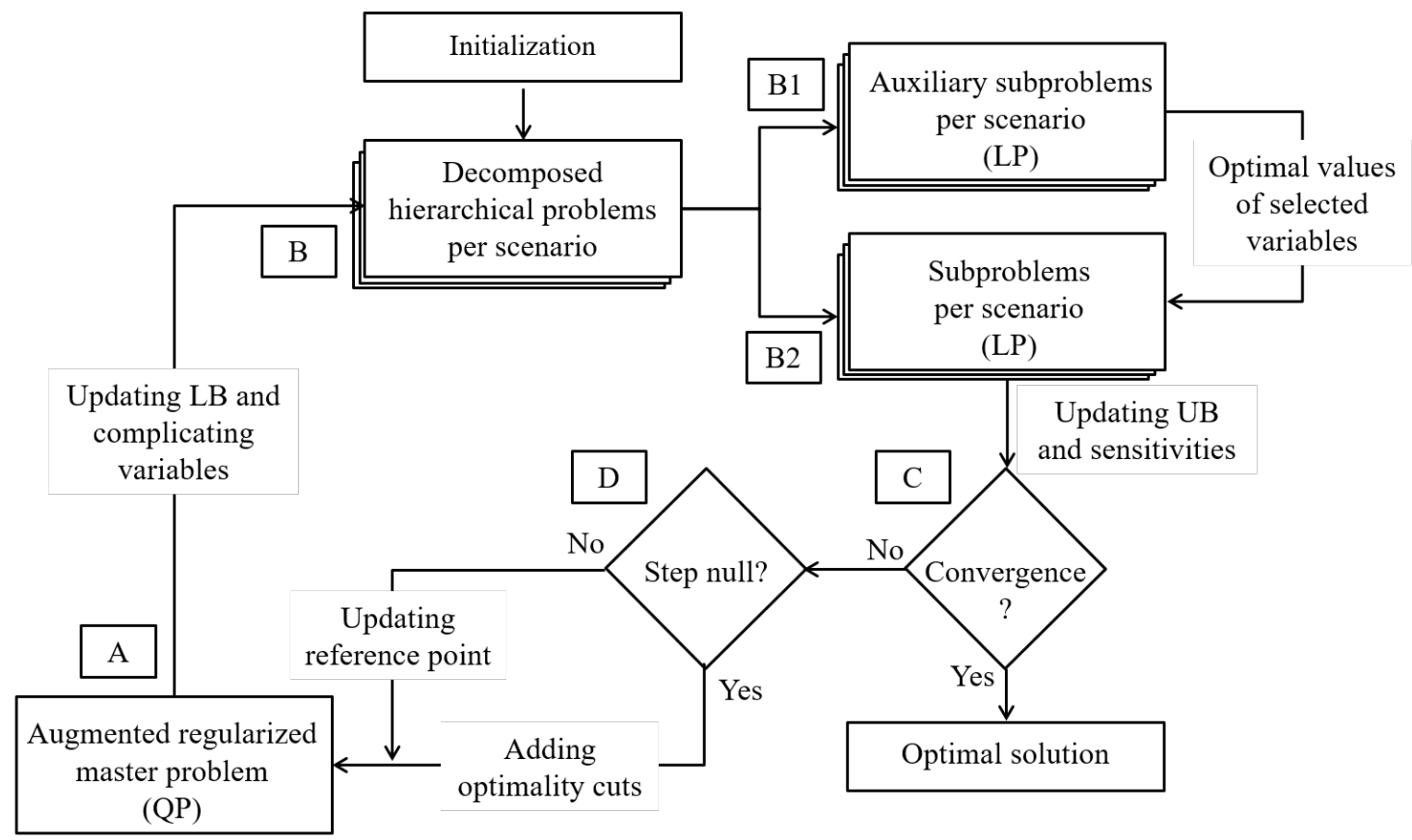

Figure 2: Augmented regularized Benders decomposition algorithm flowchart (LP: linear program; QP: quadratic program; LB: lower bound; UB: upper bound)

\subsection{Augmented Regularized Benders Decomposition Algorithm}

\subsubsection{Initialization}

In order to initialize the Benders algorithm, the original non-decomposed hierarchical optimization problem is solved for a single scenario, representing the expected value of each uncertain parameter. The initial values of the complicating variables $\Omega^{\mathrm{DA}}$ are derived from the solution of this optimization problem. The subproblems at iteration $\theta=1$ are then solved for this value of the complicating variables.

\subsubsection{Subproblems (Step B)}

At iteration $\theta \geq 1$, each subproblem $\mathrm{SUB}_{\nu}$, one per scenario $\nu \in \mathcal{X}$, is a hierarchical optimization problem. The set of optimization variables $\Omega_{\nu}^{\mathrm{SUB}}=\Omega^{\mathrm{DA}} \cup \Omega_{\nu}^{\mathrm{R}} \cup \Omega_{\nu}^{\mathrm{LL}} \cup \Xi_{\nu}^{\mathrm{LL}}$ includes the complicating variables, the redispatch variables, as well as the primal and dual lower-level variables for the corresponding scenario $\nu \in \mathcal{X}$. The objective of each subproblem $S U B_{\nu}$ is to minimize the weighted heat redispatch cost (16a) for the underlying scenario $\nu \in \mathcal{X}$, subject to heat redispatch constraints (16b), the lower-level problem $L L_{\nu}$, and the constraints fixing the complicating variables to their value updated in the master problem $(16 \mathrm{j})-(16 \mathrm{~m})$. This hierarchical optimization problem is reformulated as a single-level optimization problem using a primal-dual formulation of the lowerlevel problem, which includes the strong duality equality (16c), primal feasibility constraints (16d), 
stationarity conditions (16e)-(16h), and dual feasibility constraints (16i). This formulation is detailed in the online appendix (Mitridati et al., 2019). As a result, the subproblem $S U B_{\nu}$ can be expressed as

$$
\begin{aligned}
\min _{\Omega_{\nu}^{\mathrm{SUB}}} \pi_{\nu} \sum_{t \in \mathcal{T}} & {\left[\sum_{j \in \mathcal{I}^{\mathrm{CHP}}} \alpha_{j} \rho_{j}^{\mathrm{E}} P_{j t \nu}+\sum_{j \in \mathcal{I}^{\mathrm{E}}}\left(\alpha_{j t \nu} P_{j t \nu}+\bar{\mu}_{j t \nu} \bar{P}_{j t \nu}\right)-\lambda_{t \nu}^{\mathrm{E}} L_{t \nu}^{\mathrm{E}}\right.} \\
& \left.+\sum_{j \in \mathcal{I}^{\mathrm{CHP}} \cup \mathcal{I}^{\mathrm{HO}}}\left(\alpha_{j t \nu}^{\uparrow} Q_{j t \nu}^{\uparrow}-\alpha_{j t \nu}^{\downarrow} Q_{j t \nu}^{\downarrow}\right)\right]
\end{aligned}
$$

s.t. $(12 \mathrm{~h})-(12 \mathrm{t})$

$$
\begin{aligned}
& \sum_{j \in \mathcal{I}^{\mathrm{E}}, t \in \mathcal{T}} \tilde{\alpha}_{j t \nu}^{\mathrm{E}} P_{j t \nu}+\sum_{j \in \mathcal{I}^{\mathrm{CHP}}, t \in \mathcal{T}}\left(\underline{\alpha} P_{j t \nu}^{0}+\alpha_{j}^{\mathrm{E}} P_{j t \nu}^{+}\right)-\bar{\alpha} L_{j t \nu}^{\mathrm{HP}} \\
& =\sum_{t \in \mathcal{T}}\left[-\sum_{j \in \mathcal{I}^{\mathrm{HP}}} \bar{\mu}_{j t \nu} \underline{L}_{j}^{\mathrm{HP}}\left(Q_{j t}\right)-\sum_{j \in \mathcal{I}^{\mathrm{CHP}}} \bar{\mu}_{j t \nu}^{0} \underline{P}_{j}\left(Q_{j t}\right)\right. \\
& \left.\quad-\sum_{j \in \mathcal{I}^{\mathrm{CHP}}} \bar{\mu}_{j t \nu}\left(\bar{P}_{j}\left(Q_{j t}\right)-\underline{P}_{j}\left(Q_{j t}\right)\right)-\sum_{j \in \mathcal{I}^{\mathrm{E}}} \bar{\mu}_{j t \nu} \bar{P}_{j t \nu}+\lambda_{t \nu}^{\mathrm{E}} L_{t \nu}^{\mathrm{E}}\right] \\
& (11 \mathrm{~b})-(11 \mathrm{f}) \\
& \alpha_{j t \nu}+\bar{\mu}_{t \nu}-\underline{\mu}_{j t \nu}-\lambda_{t \nu}^{\mathrm{E}}=0, \forall j \in \mathcal{I}^{\mathrm{E}}, t \in \mathcal{T} \\
& \underline{\alpha}^{+} \bar{\mu}_{t \nu}^{0}-\underline{\mu}_{j t \nu}^{0}-\lambda_{t \nu}^{\mathrm{E}}=0, \forall j \in \mathcal{I}^{\mathrm{CHP}}, t \in \mathcal{T} \\
& \alpha_{j t \nu} \rho_{j}^{\mathrm{E}}+\bar{\mu}_{t \nu}-\underline{\mu}_{j t \nu}-\lambda_{t \nu}^{\mathrm{E}}=0, \forall j \in \mathcal{I}^{\mathrm{CHP}}, t \in \mathcal{T} \\
& -\bar{\alpha}+\bar{\mu}_{t \nu}-\underline{\mu}_{j t \nu}+\lambda_{t \nu}^{\mathrm{E}}=0, \forall j \in \mathcal{I}^{\mathrm{HP}}, t \in \mathcal{T} \\
& \underline{\mu}_{j t \nu}, \underline{\mu}_{j t \nu}^{0}, \bar{\mu}_{j t \nu}, \bar{\mu}_{j t \nu}^{0} \geq 0, \forall j \in \mathcal{I}^{\mathrm{E}} \cup \mathcal{I}^{\mathrm{CHP}} \cup \mathcal{I}^{\mathrm{HP}}, t \in \mathcal{T} \\
& Q_{j t}=Q_{j t}^{(\theta)}: \eta_{j t \nu}^{Q}, \forall j \in \mathcal{I}^{\mathrm{CHP}} \cup \mathcal{I}^{\mathrm{HP}} \cup \mathcal{I}^{\mathrm{HO}}, t \in \mathcal{T} \\
& S_{j t}=S_{j t}^{(\theta)}: \eta_{j t \nu}^{S}, \forall j \in \mathcal{I}^{\mathrm{HS}}, t \in \mathcal{T} \\
& Q_{j t}^{+}=Q_{j t}^{+(\theta)}: \eta_{j t \nu}^{Q^{+}}, \forall j \in \mathcal{I}^{\mathrm{HS}}, t \in \mathcal{T} \\
& Q_{j t}^{-}=Q_{j t}^{-(\theta)}: \eta_{j t \nu}^{Q^{-}}, \forall j \in \mathcal{I}^{\mathrm{HS}}, t \in \mathcal{T} .
\end{aligned}
$$

Due to the bilinear terms $\bar{\mu}_{j t \nu}^{0} \underline{P}_{j}\left(Q_{j t}\right), \bar{\mu}_{j t \nu}\left(\bar{P}_{j}\left(Q_{j t}\right)-\underline{P}_{j}\left(Q_{j t}\right)\right)$, and $\bar{\mu}_{j t \nu} \underline{L}_{j}^{\mathrm{HP}}\left(Q_{j t}\right)$ in $(16 \mathrm{c})$, the subproblems are non-convex. Therefore, this subproblem is solved in two steps. Each bilinear term is in fact a product of a complicating variable (say $Q_{j t}$ ) and a dual variable $\left(\right.$ say $\bar{\mu}_{j t \nu}$ ). At a given iteration $\theta$, in order to linearize these terms, each subproblem is solved in two steps. We first solve a linear auxiliary subproblem $\left(\operatorname{aux}-\mathrm{SUB}_{\nu}\right)$, in which the complicating variables $Q_{j t}$ are treated as parameters and fixed to the values $Q_{j t}^{(\theta)}$ obtained in the master problem, and not as variables fixed to given values (step B1). These auxiliary subproblems provide the optimal values for dual variables $\bar{\mu}_{j t \nu}$, but do not give sensitivities required for generating cuts. In the second step, the dual variables $\bar{\mu}_{j t \nu}$ are treated as parameters and fixed to those values $\bar{\mu}_{j t \nu}^{(\theta)}$ obtained in step B1, and subproblems $\mathrm{SUB}_{\nu}$ treat complicating variables $Q_{j t}$ as variables. However, the fixing constraints enforce the complicating variables $Q_{j t}$ to take the values $Q_{j t}^{(\theta)}$ coming from the master problem. The dual variables of the fixing constraints $(16 \mathrm{j})-(16 \mathrm{~m}), \eta_{j t \nu}^{Q}, \eta_{j t \nu}^{S}, \eta_{j t \nu}^{Q^{+}}, \eta_{j t \nu}^{Q^{-}}$, provide 
sensitivities with respect to the complicating variables (step B2). These sensitivities are derived from the subproblems at each iteration in order to generate optimality cuts that further constrain the master problem in the following iterations.

\subsubsection{Augmented Regularized Master Problem (Step A)}

At each iteration the master problem seeks to update the set of complicating variables $\Omega^{\mathrm{DA}}$, using information from the previous iterations of subproblems. For notational simplicity, we introduce the vector of complicating variables $X=\left[Q_{j t}, S_{j t}, Q_{j t}^{+}, Q_{j t}^{-}\right]^{\top}$. The set of optimization variables $\Omega^{\mathrm{MP}}=\Omega^{\mathrm{DA}} \cup\{\beta\}$ includes the complicating variables, and the auxiliary variable $\beta$ representing the current linear estimate of the objective function of the subproblems. The master problem aims at minimizing the day-ahead heat cost plus the current linear estimate of the objective function of the subproblems, i.e., $\beta$, while the quadratic penalization term $\frac{\left\|X-X^{(\mathrm{ref})}\right\|_{2}}{\tau^{(\mathrm{ref})}}$ in (17a) ensures that the solutions remain close to the current reference point $X^{(\mathrm{ref})}$. The update of the reference point is detailed in Section 4.2.4. Efficient heuristic methods have been introduced to update the penalization parameter $\tau^{(\mathrm{ref})}$, that result in an accelerated convergence of the bundle method (Rey \& Sagastizábal, 2002; Bonnans et al., 2006). The constraints of the master problem include all constraints from the non-decomposed problem involving solely the complicating variables (17b). Additionally, two sets of cuts are added to the master problem at each iteration. First, the optimality cuts $(17 \mathrm{c})$ are linear under-estimators of the objective function of the subproblems at previous iterations $k<\theta$. At each iteration $\theta>1$ a single optimality cut is added, and (17d) enforces a lower bound for the initial iteration $\theta=1$. Second, the set of auxiliary cuts $(17 \mathrm{e})-(17 \mathrm{~g})$ represent some of the primal constraints of the subproblems at previous iteration $\theta$. These cuts create an additional feedback from the subproblems which guides the master problem towards an optimal solution. As a result, at iteration $\theta+1$, the following master problem is solved to update the values of the complicating variables of the Benders algorithm:

$$
\begin{aligned}
& \min _{\Omega^{\mathrm{MP}}} \sum_{\nu \in \mathcal{X}, t \in \mathcal{T}}\left[\sum_{j \in \mathcal{I}^{\mathrm{HO}}} \alpha_{j} Q_{j t}+\sum_{j \in \mathcal{I}^{\mathrm{CHP}}} \alpha_{j} \rho_{j}^{\mathrm{H}} Q_{j t}\right]+\beta+\frac{\left\|X-X^{(\mathrm{ref})}\right\|_{2}}{\tau^{(\mathrm{ref})}} \\
& \text { s.t. }(12 \mathrm{~b})-(12 \mathrm{f}) \\
& \beta \geq \sum_{\nu \in \mathcal{X}, t \in \mathcal{T}}\left[z_{\nu}^{\mathrm{SUB},(k)}+\sum_{j \in \mathcal{I}^{\mathrm{HO}} \cup \mathcal{I}^{\mathrm{CHP}} \cup \mathcal{I}^{\mathrm{HP}}} \eta_{j t \nu}^{Q,(k)}\left(Q_{j t}^{(k)}-Q_{j t}\right)+\sum_{j \in \mathcal{I}^{\mathrm{HS}}}\left(\eta_{j t \nu}^{Q^{+},(k)}\left(Q_{j t}^{+,(k)}-Q_{j t}^{+}\right)\right.\right. \\
& \left.\left.+\eta_{j t \nu}^{Q^{-},(k)}\left(Q_{j t}^{-,(k)}-Q_{j t}^{-}\right)+\eta_{j t \nu}^{S,(k)}\left(S_{j t}^{(k)}-S_{j t}\right)\right)\right], \forall k \in[1, \ldots, \theta] \\
& \beta \geq-M \\
& \sum_{\nu \in \mathcal{X}} \pi_{\nu} P_{j t \nu}^{(\theta)} \geq r_{j}\left(Q_{j t}+\sum_{\nu \in \mathcal{X}} \pi_{\nu}\left(Q_{j t \nu}^{\uparrow,(\theta)}-Q_{j t \nu}^{\downarrow,(\theta)}\right)\right), \forall j \in \mathcal{I}^{\mathrm{CHP}}, t \in \mathcal{T} \\
& \rho_{j}^{\mathrm{E}} \sum_{\nu \in \mathcal{X}} \pi_{\nu} P_{j t \nu}^{(\theta)}+\rho_{j}^{\mathrm{H}}\left(Q_{j t}+\sum_{\nu \in \mathcal{X}} \pi_{\nu}\left(Q_{j t \nu}^{\uparrow,(\theta)}-Q_{j t \nu}^{\downarrow,(\theta)}\right)\right) \leq \bar{F}_{j}, \forall j \in \mathcal{I}^{\mathrm{CHP}}, t \in \mathcal{T} \\
& Q_{j t}+\sum_{\nu \in \mathcal{X}} \pi_{\nu}\left(Q_{j t \nu}^{\uparrow,(\theta)}-Q_{j t \nu}^{\downarrow,(\theta)}\right) \geq \operatorname{COP}_{j} \sum_{\nu \in \mathcal{X}} \pi_{\nu} L_{j t \nu}^{\mathrm{HP},(\theta)}, \forall j \in \mathcal{I}^{\mathrm{HP}}, t \in \mathcal{T} .
\end{aligned}
$$




\subsubsection{Bounds and Reference Point Update (Step D)}

At each iteration $\theta+1$, the solutions of the subproblems provide a new upper-bound of the objective value, and a reference point for the Benders algorithm. If the decrease in the expected objective value of the subproblems is significant, then the upper-bound is updated, in which case the step is called non-null. Otherwise, it remains at the same value, in which case the step is called null. More precisely, for a given parameter $0<m<1$,

$$
\mathrm{UB}^{(\theta+1)}=\left\{\begin{array}{l}
\mathrm{UB}^{(\theta)}, \text { if } z^{\mathrm{MP},(\theta+1)}+\sum_{\nu \in \mathcal{X}} z_{\nu}^{\mathrm{SUB},(\theta+1)} \geq \mathrm{ub}^{(\theta)}-m\left(\mathrm{UB}^{(\theta)}-\mathrm{LB}^{(\theta)}\right) \\
z^{\mathrm{MP},(\theta+1)}+\sum_{\nu} z_{\nu}^{\mathrm{SUB},(\theta+1)}, \text { otherwise. }
\end{array}\right.
$$

Similarly, if the step is non-null the value of the current reference point of the Benders algorithm is updated, such that

$$
X^{(\mathrm{ref})}=\left[Q_{j t}^{(\theta+1)}, S_{j t}^{(\theta+1)}, Q_{j t}^{+,(\theta+1)}, Q_{j t}^{-,(\theta+1)}\right]^{\top} .
$$

The choice of the parameter $m$ influences the size of the Benders steps, and hence the frequency of the update of the upper-bound. However, for convex problems the bundle method has been proved to converge for any value $0<m<1$ (Rey \& Sagastizábal, 2002; Bonnans et al., 2006). Additionally, the objective value of the master problem $z^{\mathrm{MP},(\theta+1)}$ provides a lower-bound for the solution of the Benders algorithm, such that $\mathrm{LB}^{(\theta+1)}=z^{\mathrm{MP},(\theta+1)}$.

\subsubsection{Convergence Check (Step C)}

Finally, the Benders decomposition algorithm has converged when the upper and lower bounds have converged. More precisely, for an arbitrarily small tolerance parameter $\epsilon>0$, the algorithm has converged when $\left|\mathrm{UB}^{(\theta)}-\mathrm{LB}^{(\theta)}\right| \leq \epsilon$.

\subsection{Convergence Issues}

Benders decomposition algorithms are assured to converge as long as the objective function of the original problem is convex with respect to the complicating variables. The hierarchical optimization problem considered is non-convex, but an increasing number of scenarios results in a smoother and asymptotically convex objective function with respect to complicating variables. Bertsekas \& Sandell (1982) analyze the theoretical foundation of this behavior. Hence, by increasing the number of scenarios considered a successful implementation of Benders decomposition is possible.

In practice, although the proposed Benders algorithm is not mathematically guaranteed to converge, convergence to the optimal solution of the hierarchical optimization problem (12) was observed on a simple motivating example presented in the online appendix (Mitridati et al., 2019) for any number of scenarios greater than eight. Convergence was validated by comparing the solutions of original and decomposed models for those cases that have a tractable solution for the original non-decomposed model.

\section{Numerical Example}

This section compares the proposed electricity-aware market framework to two benchmarks, namely a sequential decoupled market framework, inspired by the current Danish market framework (Varmelast, 2018; Nordpool, 2019), and an integrated market, inspired by Chen et al. (2015). The mathematical formulations of the sequential and integrated market-clearing procedures are detailed 
in the online appendix (Mitridati et al., 2019). These three market frameworks for integrated energy systems are compared in terms of the total operational cost of the system for varying values of the wind power penetration. In order to model accurately the uncertainty from the power system, a large number of scenarios is considered. The electricity-aware heat market-clearing problem is solved using the Benders algorithm described in Section 4. The simulations are implemented in Python 3.5, using Gurobi 7.0.2 solver, on a Processor Intel Core i5-6200U CPU @ $2.30 \mathrm{GHz}(8.00$ GB RAM). All codes and input data are available online in Mitridati et al. (2019).

\subsection{Description of Simulation Setup and Experiments}

We consider a modified version of the 24-bus IEEE reliability test system. It consists of seven thermal power plants, six wind farms, four extraction CHPs, and one heat-only unit. Data for the power system is derived from Ordoudis et al. (2016). The district heating system comprises four CHPs, a heat-only peak unit with a capacity of $8000 \mathrm{MW}$ and three heat storage tanks with a total storage capacity of $450 \mathrm{MWh}$. The four CHPs account for $1,300 \mathrm{MW}$ of the total $2,100 \mathrm{MW}$ installed capacity of thermal generation in the district heating system, and for 1,107MW of the total 2, 864MW in the power system. Data for the technical characteristics of CHPs, heat storage units, and heat loads are representative of the greater Copenhagen area, and derived from Madsen (2015) and Zugno et al. (2016). Furthermore, to reflect the evolution of the Danish energy system, three large-scale HPs with a total capacity of 750MW are installed (Meibom et al., 2007; Bach et al., 2016).

Sources of uncertainty in the day-ahead electricity market, namely wind production, electricity loads and supply functions, are considered independent. Wind power uncertainty is modeled by a set of scenarios with temporal and spatial correlation as introduced by Bukhsh et al. (2016) and available in Bukhsh (2015). The original dataset represents real scenarios of wind production factors (between 0 and 1) in Denmark. The interested reader is referred to Pinson (2013) for a detailed overview of the challenges related to short-term wind power forecasting and scenario generation. Electricity load scenarios are derived from Nordpool market data for January 2018, available in Nordpool (2018), and resized to fit into the 24-bus IEEE reliability test system. The average electricity load factors between 0 and 1 are derived from historical data by computing an average week-day hourly load, and normalizing it between 0 and 1 based on the observed average daily peakload and base-load. The standard deviation of electricity loads is computed similarly for each hour of a week-day. Scenarios of load factors are then derived at each hour using independent Normal distribution centered around the average load factors and using the standard deviation. Finally, the load factors are resized linearly between the minimum and maximum daily load. These bounds are derived from the updated 24-bus IEEE test system presented in Ordoudis et al. (2016). As supply functions in electricity markets are seldom observable due to privacy concerns, forecasting supply functions is challenging and requires advanced modeling tools (Weber, 2006; Chen et al., 2019). For the sake of simplicity, scenarios of price bids $\tilde{\alpha}_{j t \nu}^{\mathrm{E}}$ for each electricity producer are generated from marginal costs in the 24-bus IEEE reliability test system, using independent Normal distributions for each hour of the day. Mitridati \& Pinson (2018) provide a more advanced model for characterizing the uncertainty of supply functions, solely based on historical observations of spot prices and energy traded in the Nordpool electricity market. The proposed Bayesian inference approach in Mitridati \& Pinson (2018) is out of the scope of this study. This numerical example is detailed in the online appendix (Mitridati et al., 2019). Despite the lack of available data for real-life integrated heat and electricity systems, this numerical example is designed to be representative of the current and future energy mix in the Danish energy system (Lund et al., 2010; Meibom et al., 2013). 
The following analysis compares the performance of the three market frameworks for integrated energy systems over a 24-hour scheduling horizon. This analysis is carried out for different values of installed wind capacity, ranging from $450 \mathrm{MW}$ to $1,800 \mathrm{MW}$, i.e., between $32 \%$ and $138 \%$ of the peak demand.

The performance of the proposed stochastic hierarchical optimization problem depends greatly on the accurate description of the uncertainty in the power system. However, the computational complexity of this model increases with the number of scenarios. Therefore, an appropriate tradeoff between uncertainty representation and computational complexity must be found. Each source of uncertainty in the day-ahead electricity market, namely wind production, electricity loads and supply functions, is modeled by 15 independent scenarios (i.e., 3, 375 scenarios in total). For each independent source of uncertainty, a scenario reduction technique is implemented to merge similar scenarios (Gabriel et al., 2009; Morales et al., 2009). Figure 3 illustrates the mean value and standard deviation of the objective value of the electricity-aware heat market clearing, and the CPU time of the proposed Benders algorithm, depending on the number of scenarios selected. It shows that the proposed Benders algorithm provides a tractable solution method for the proposed stochastic hierarchical optimization problem, whereas the non-decomposed MILP formulation is computationally intractable for this case study with a number of scenarios higher than two. As a result, the convergence of the Benders decomposition algorithm cannot be directly validated for those cases. However, as discussed in Section 4.3, increasing the number of scenarios is expected to facilitate a successful implementation of the proposed Benders decomposition algorithm. Additionally, both mean value and standard deviation of the objective value remain stable for a number of scenarios higher than 216 . This implies that 216 scenarios are sufficient to capture the uncertainty of the original set of 3,375 scenarios. Thus, 216 scenarios, i.e., 6 scenarios per source of uncertainty, are selected for this study, which provide a trade-off between uncertainty representation and computational complexity. The same probability is assigned to each scenario.
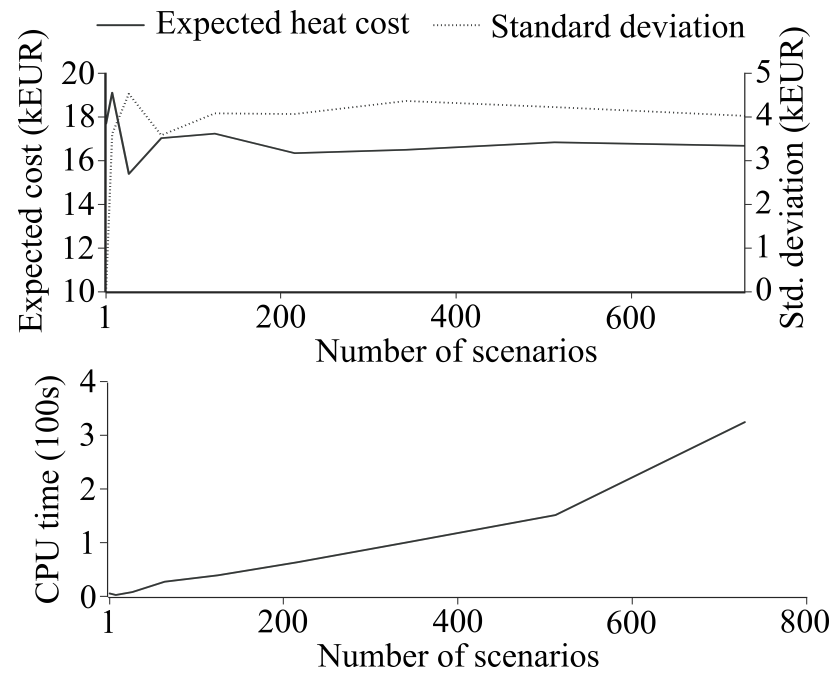

Figure 3: Electricity-aware heat market clearing (Benders algorithm) - expected cost and standard deviation of heat cost (top plot), and CPU time (bottom plot) as a function of the number of scenarios

These 216 representative scenarios are used to describe the sources of uncertainty in the electricityaware heat market clearing in problem (12), which is solved using the augmented regularized Ben- 
ders algorithm introduced in Section 4. As this simulation is performed in-sample, the day-ahead electricity market (step 2) and heat redispatch (step 3) are then cleared for the same scenarios. Once the sequential heat market framework is cleared, the day-ahead electricity market (step 2) and heat redispatch (step 3) are cleared for all scenarios. In the integrated market framework, heat and electricity are simultaneously dispatched for each scenario.

Additionally, in the sequential heat market clearing, CHPs and HPs require forecast electricity prices $\hat{\lambda}_{t}^{\mathrm{E}}$ as input parameters to compute their expected heat marginal cost $\alpha_{j}^{\mathrm{H}}\left(\hat{\lambda}_{t}^{\mathrm{E}}\right)$ in $(9)-(10)$. In order to make a fair comparison between the sequential market-clearing model and the two other models, it is assumed that all heat market participants use the same electricity price forecasts. In practice, heat market participants may use different electricity price forecasts based on their individual information, which may distort the merit order in the heat market clearing. Therefore, it is expected that relaxing this assumption would introduce additional inefficiencies in the sequential heat market clearing. For that purpose, we first simulate the heat and electricity dispatch (steps 1 and 2) in the electricity-aware market framework with a single trajectory, representing the expected value of the original 3,375 scenarios. The resulting spot prices are then used as input parameters to compute heat marginal costs in the sequential market clearing. Additionally, the resulting dayahead heat dispatch is used to initialize the Benders algorithm described in Section 4.

\subsection{Impact of Market Coordination on Cost-Effective Operation of Heat and Electricity Systems}

Figure 4 depicts the profile of the expected heat and power system costs across the three aforementioned market frameworks for different values of installed wind capacity. The expected heat cost is computed as the summation of the day-ahead heat production cost and the redispatch cost in (12) accounted for all scenarios. In the integrated market, the redispatch cost is always zero. The expected electricity cost is computed as CHPs and electricity generators' production costs accounted for all scenarios. This method is the most common way to allocate CHPs and HPs' costs in practice. However, the expected total system cost, which is computed as the production cost of the integrated energy system accounted for all scenarios, is not equal to the sum of expected heat and electricity costs. The left-hand side plot of Figure 4 illustrates that the electricity production

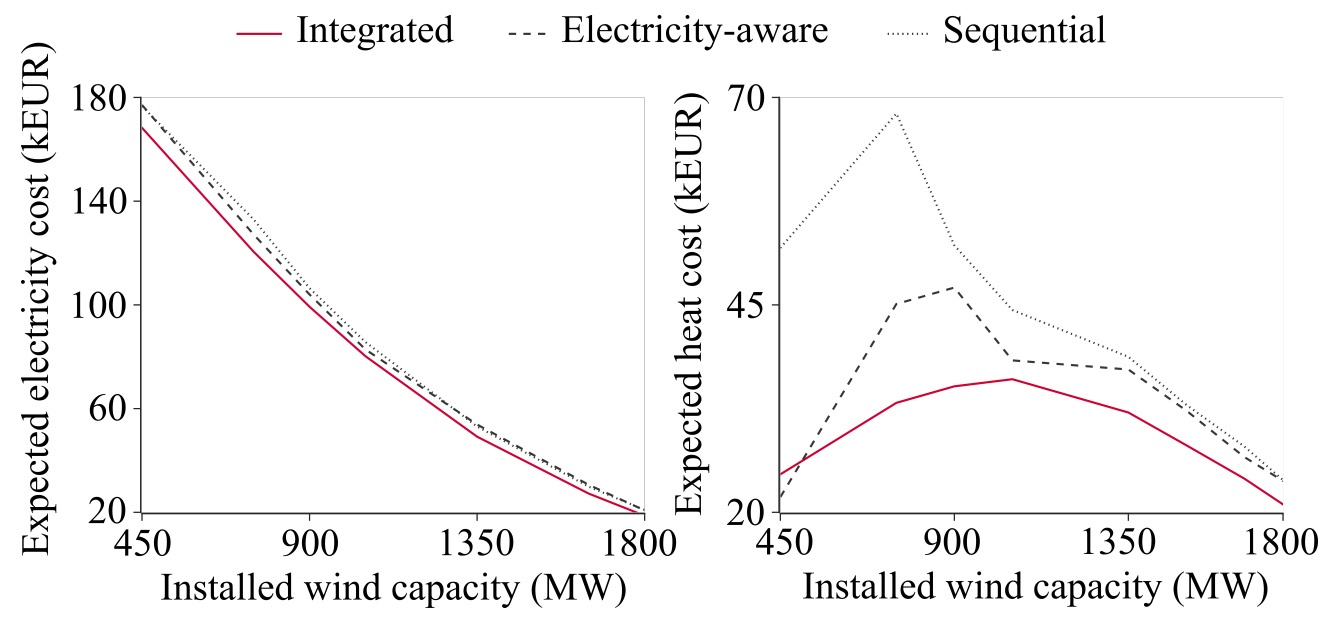

Figure 4: Expected in-sample electricity (left-hand side plot) and heat (right-hand side plot) production costs across the three market frameworks: sequential, integrated, and electricity-aware (Benders algorithm)

cost decreases steadily in the integrated market framework, whereas in the right-hand side plot the 
heat production cost remains relatively stable. A slight increase of the heat production cost can be noticed for installed wind capacities ranging from $450 \mathrm{MW}$ to $1,050 \mathrm{MW}$, due to a decrease in average electricity prices, resulting in an increase in CHPs' heat marginal costs. For higher values of the installed wind capacity, HPs become more profitable and the heat system benefits from the decrease in electricity prices. Heat costs in the sequential and electricity-aware market frameworks follow a similar pattern, but for wind capacities between $450 \mathrm{MW}$ and 1,050MW the increase is more pronounced.

The sequential heat market is myopic to the impact of CHPs and HPs on electricity prices, and thus to their impact on heat marginal costs and the merit order in the heat market. As a result, the sequential market framework results in the highest heat production cost. As expected, the electricity-aware market framework always achieves a lower expected heat production cost compared to that in the sequential one. This improvement is achieved by modeling endogenously the participation of CHPs and HPs in the power system, and accounting for the impact of the sources of uncertainty on the redispatch cost. However, there is no guarantee for the improvement of the expected electricity and total system costs.

In the sequential and electricity-aware market frameworks, the day-ahead heat dispatch of CHPs and HPs, and therefore their capability for producing electricity are fixed before the electricity market clearing. This heat-driven approach limits the flexibility of CHPs and HPs in the electricity market, resulting in a sharp increase in expected electricity cost with respect to that in the integrated market, as presented in Table 1.

Table 1: Difference in expected electricity, heat, and total system costs across the sequential and electricity-aware market frameworks with respect to those in the integrated market framework for different values of installed wind capacity (expressed in \%, with the integrated market framework as reference)

\begin{tabular}{lccccccc}
\hline & & \multicolumn{3}{c}{ Sequential } & \multicolumn{3}{c}{ Electricity-aware } \\
Wind capacity & $(\mathrm{MW})$ & 450 & 900 & 1,800 & 450 & 900 & 1,800 \\
\hline Electricity cost & $(\%)$ & 5.0 & 7.2 & 11.9 & 5.2 & 4.8 & 11.1 \\
Heat cost & $(\%)$ & 111.0 & 48.3 & 12.6 & -11.1 & 33.9 & 12.3 \\
Total system cost & $(\%)$ & 7.8 & 10.6 & 13.3 & 3.5 & 6.8 & 9.8 \\
\hline
\end{tabular}

As the integrated market seeks to minimize the total system cost for each scenario, contrary to the two other market frameworks, it is guaranteed to always provide a lower bound for the expected total system cost. However, there is no such a guarantee regarding the expected electricity and heat production costs. Figure 5 depicts the profile of the expected total system cost across the three market frameworks for different values of installed wind capacity. As expected, the integrated market framework consistently achieves the lowest expected total system cost for all values of wind penetration. The sequential market framework, due to its lack of foresight on the impact of the heat market outcomes on the power system, results in a sharp increase in expected total system costs. As seen in Table 1, the relative gap in expected total system costs between the sequential and integrated market frameworks widens when wind penetration increases. This is mostly driven by the growing gap in expected electricity cost, and by the increasing heat redispatch cost. Interestingly, the electricity-aware market framework provides a significant improvement in expected total system cost compared to the sequential one, as exhibited in Table 1. Even though the electricity-aware market framework improves the coordination between heat and electricity systems compared to the sequential one, it still provides a limited flexibility due to the sequential dispatch of heat and electricity systems. On the contrary, in the integrated market framework heat 

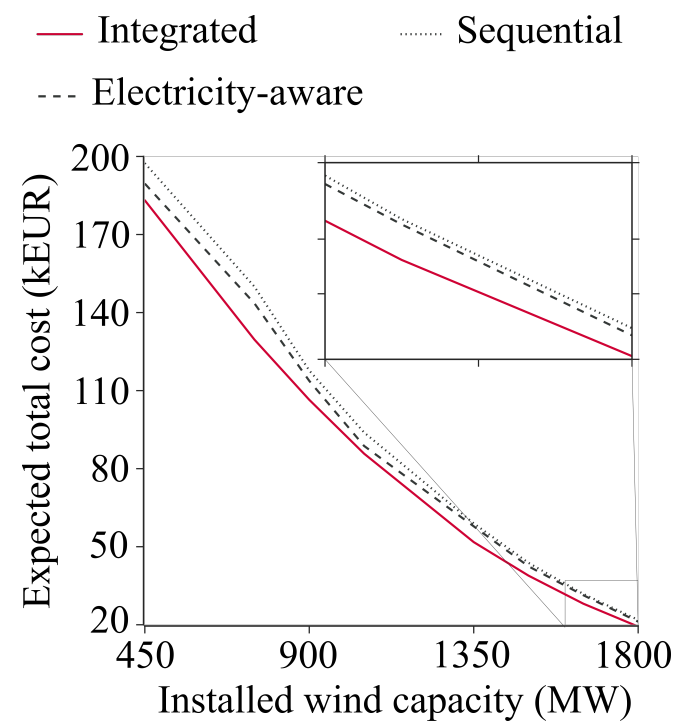

Figure 5: Expected in-sample total system cost across the three market frameworks: sequential, integrated, and electricity-aware (Benders algorithm).

and electricity systems are dispatched simultaneously, allowing the market operator to fully exploit the operational flexibility of CHPs and HPs. Therefore, when the level of uncertainty from wind production increases, the relative gap in expected total system costs between the electricity-aware and integrated market frameworks grows, as shown in Table 1.

\subsection{Impact of Unseen Scenarios}

This subsection presents an out-of-sample analysis to provide a more rigorous comparison of the performance of the three market frameworks against unseen scenarios. To this purpose, 1,000 new scenarios are generated from the same distributions. Wind and electricity demand scenarios are presented in the online appendix (Mitridati et al., 2019). Fixing the day-ahead heat dispatch to those obtained in the in-sample simulations, we run a day-ahead electricity market and then a heat redispatch, as well as an integrated heat and electricity market for each out-of-sample scenario. Figures 6 and 7 depict how the expected heat, electricity and total system costs evolve considering out-of-sample scenarios for increased wind capacity.

As expected, the expected heat, electricity and total system costs achieved with the sequential and electricity-aware market frameworks increase compared to those in the in-sample simulation, due to the impact of unseen scenarios on expected electricity and heat redispatch costs. The integrated market still provides a lower bound for the expected total system cost, while the electricityaware market framework consistently achieves a lower expected heat system cost compared to that in the sequential one. In fact, the relative gap in expected heat cost between the electricity-aware and sequential market frameworks increases compared to the in-sample simulation, as exhibited in Table 2. This is achieved by a better representation of the sources of uncertainty and their impact on redispatch costs in the electricity-aware heat market. Furthermore, the electricity-aware market framework still achieves a significant improvement in the expected total system cost compared to the sequential one. However, the relative gap in expected total system cost between electricityaware and sequential market frameworks decreases compared to that in the in-sample simulations, as presented in Table 2 . 

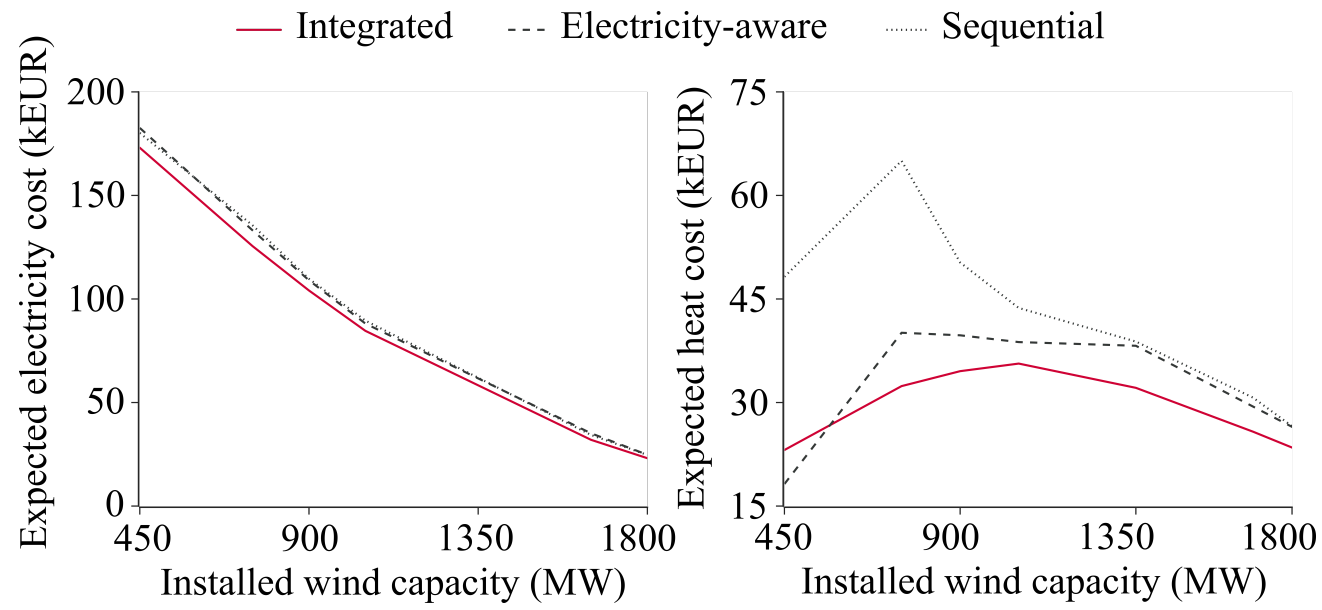

Figure 6: Expected out-of-sample electricity (left-hand side plot) and heat (right-hand side plot) production costs across the three market frameworks: sequential, integrated, and electricity-aware (Benders algorithm)

\section{— Integrated \\ Sequential \\ -.- Electricity-aware}

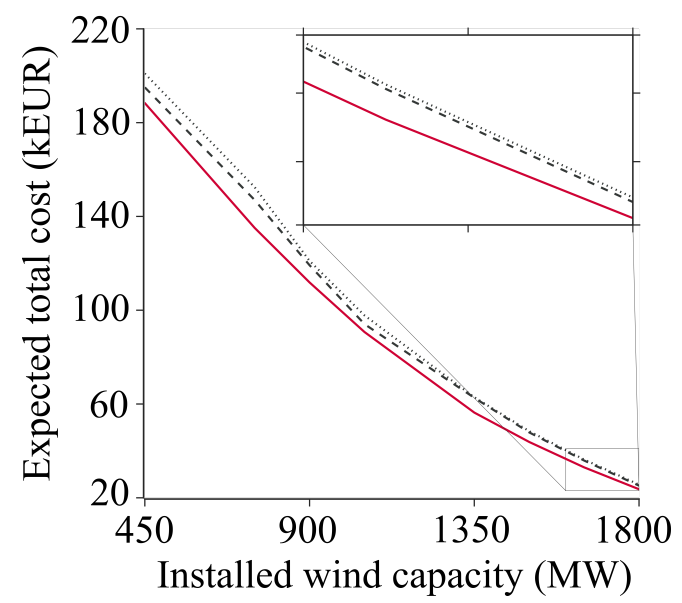

Figure 7: Expected out-of-sample total system costs across the three market frameworks: sequential, integrated, and electricity-aware (Benders algorithm)

Table 2: Difference in expected electricity, heat, and total system costs in the electricity-aware market framework with respect to those in the sequential market framework for different values of installed wind capacity, with in-sample and out-of-sample scenarios (expressed in \%, with the sequential market framework as reference)

\begin{tabular}{lccccccc}
\hline & & \multicolumn{3}{c}{ In-sample } & \multicolumn{3}{c}{ Out-of-sample } \\
Wind capacity & $(\mathrm{MW})$ & 450 & 900 & 1,800 & 450 & 900 & 1,800 \\
\hline Electricity cost & $(\%)$ & +0.3 & -2.3 & -0.7 & +1.4 & -0.7 & +0.4 \\
Heat cost & $(\%)$ & -57.9 & -9.7 & -0.2 & -62.3 & -26.6 & -0.3 \\
Total system cost & $(\%)$ & -4.0 & -3.4 & -3.0 & -2.9 & -1.5 & -1.7 \\
\hline
\end{tabular}




\subsection{Performance Bounds of Electricity-Aware Heat Market-Clearing Model}

The results presented in Sections 5.2 and 5.3 assume that the electricity loads $L_{t}^{E}$ and aggregate supply curve $\alpha_{j t}^{E}$ in the electricity market are distributed according to the independent Normal distributions $\mathcal{N}\left(\mu_{t}^{L^{E}},\left(\sigma_{t}^{L^{E}}\right)^{2}\right)$ and $\mathcal{N}\left(\mu_{j t}^{\alpha^{E}},\left(\sigma_{j t}^{L^{E}}\right)^{2}\right)$, respectively. As these distributions are assumed to be independent and uncorrelated, the guessed joint distribution is a Normal distribution $\mathcal{N}_{\mathcal{G}}\left(\boldsymbol{\mu}, \boldsymbol{\sigma}^{2}\right)$ with mean $\boldsymbol{\mu}=\left[\mu_{1}^{L^{E}}, . ., \mu_{T}^{L^{E}}, \mu_{1,1}^{\alpha^{E}}, \ldots, \mu_{J, T}^{\alpha^{E}}\right]$ and diagonal covariance matrix, and with diagonal vector $\boldsymbol{\sigma}^{\mathbf{2}}=\left[\left(\sigma_{1}^{L^{E}}\right)^{2}, . .,\left(\sigma_{T}^{L^{E}}\right)^{2},\left(\sigma_{1,1}^{\alpha^{E}}\right)^{2}, \ldots,\left(\sigma_{J, T}^{\alpha^{E}}\right)^{2}\right]$.

As pointed out in Maggioni et al. (2019) and Pantuso et al. (2015), different parameters can influence the accuracy of multi-stage stochastic programs, such as shape, support, and moments of the right and guessed distributions. This subsection studies the impact of the error in the first- and second-order moments, i.e., mean and variance, of the guessed distribution $\mathcal{N}_{\mathcal{G}}\left(\boldsymbol{\mu}, \boldsymbol{\sigma}^{2}\right)$. However, the assumption that the distributions are uncorrelated and independent is maintained.

In order to model errors in the guessed distributions, the mean values of the guessed distributions of electricity load factors are perturbed uniformly by an error factor $\eta^{\mu}$ ranging from 0.5 to 1.5, i.e., assuming up to $50 \%$ error in the guessed mean of distributions. The variance of the guessed Normal distribution of the electricity load factors is then perturbed by an error factor $\eta_{\left(\sigma^{L}\right)^{2}}$ ranging from 0.5 to 1.5. Similarly, the mean and variance of the guessed Normal distribution of the aggregated supply curve are perturbed by error factors $\eta_{\mu^{\alpha^{E}}}$ and $\eta_{\left(\sigma^{\alpha^{E}}\right)^{2}}$, respectively. As each error factor takes 21 values between 0.5 and 1.5 while all other error factors are fixed to 1.0, this process results in 81 distinct combinations of these factors. Each combination of the error factors $\eta_{\mu^{L^{E}}}, \eta_{\left(\sigma^{L^{E}}\right)^{2}}, \eta_{\mu^{\alpha^{E}}}$, and $\eta_{\left(\sigma^{\alpha^{E}}\right)^{2}}$, corresponds to a right joint Normal distribution $\mathcal{N}_{\mathcal{R}}\left(\boldsymbol{\eta}_{\boldsymbol{\mu}} \boldsymbol{\mu}, \boldsymbol{\eta}_{\boldsymbol{\sigma}^{2}} \boldsymbol{\sigma}^{\mathbf{2}}\right)$ of mean $\boldsymbol{\eta}_{\boldsymbol{\mu}} \boldsymbol{\mu}=$ $\left[\eta_{\mu^{L^{E}}} \mu_{1}^{L^{E}}, . ., \eta_{\mu^{L^{E}}} \mu_{T}^{L^{E}}, \eta_{\mu^{\alpha^{E}}} \mu_{1,1}^{\alpha^{E}}, \ldots, \eta_{\mu^{\alpha^{E}}} \mu_{J, T}^{\alpha^{E}}\right]$ and diagonal covariance matrix, with diagonal vector $\boldsymbol{\eta}_{\boldsymbol{\sigma}^{\mathbf{2}}} \boldsymbol{\sigma}^{\mathbf{2}}=\left[\eta_{\left(\sigma^{L^{E}}\right)^{2}}\left(\sigma_{1}^{L^{E}}\right)^{2}, . ., \eta_{\left(\sigma^{L^{E}}\right)^{2}}\left(\sigma_{T}^{L^{E}}\right)^{2}, \eta_{\left(\sigma^{\alpha^{E}}\right)^{2}}\left(\sigma_{1,1}^{\alpha^{E}}\right)^{2}, \ldots, \eta_{\left(\sigma^{\alpha^{E}}\right)^{2}}\left(\sigma_{J, T}^{\alpha^{E}}\right)^{2}\right]$. Additionally, 1, 000 scenarios are generated from each right distribution in order to solve the first-stage electricityaware heat market and second-stage electricity market and heat redispatch problems. The optimal first-stage heat dispatch from the electricity-aware heat market clearing using the guessed Normal distribution $\mathcal{N}_{\mathcal{G}}\left(\boldsymbol{\mu}, \boldsymbol{\sigma}^{2}\right)$ is denoted as $Q^{\mathcal{G}^{*}}$. Additionally, the optimal first-stage heat dispatch using the right distribution $\mathcal{N}_{\mathcal{R}}\left(\boldsymbol{\eta}_{\boldsymbol{\mu}} \boldsymbol{\mu}, \boldsymbol{\eta}_{\sigma^{2}} \boldsymbol{\sigma}^{\mathbf{2}}\right)$ is denoted as $Q^{\mathcal{N}^{*}}\left(\boldsymbol{\eta}_{\boldsymbol{\mu}}, \boldsymbol{\eta}_{\boldsymbol{\sigma}^{2}}\right)$. Following the approach proposed in Maggioni et al. (2019), the following measures are defined in order to represent the impact of considering the wrong guessed distribution:

1. The expectation of the guessed distribution $\mathcal{N}_{\mathcal{G}}\left(\boldsymbol{\mu}, \boldsymbol{\sigma}^{2}\right)$ is defined as the expected out-of-sample system cost computed using 1,000 scenarios generated from the right distribution, and given the fixed first-stage decisions $Q^{\mathcal{G}^{*}}$. It is defined as

$$
\mathbb{E} \mathcal{G}\left(\boldsymbol{\eta}_{\boldsymbol{\mu}}, \boldsymbol{\eta}_{\boldsymbol{\sigma}^{2}}\right)=\mathbb{E}_{\mathcal{N}_{\mathcal{R}}\left(\boldsymbol{\eta}_{\boldsymbol{\mu}} \boldsymbol{\mu}, \boldsymbol{\eta}_{\boldsymbol{\sigma}^{2}} \sigma^{2}\right)} \mathcal{C}\left(Q^{\mathcal{G}^{*}}\right)
$$

2. The expectation of the right distribution $\mathcal{N}_{\mathcal{R}}\left(\boldsymbol{\eta}_{\boldsymbol{\mu}} \boldsymbol{\mu}, \boldsymbol{\eta}_{\boldsymbol{\sigma}^{\mathbf{2}}} \boldsymbol{\sigma}^{\mathbf{2}}\right)$ is defined as the expected insample system cost computed using 1,000 scenarios generated from the right distribution, and given the fixed first-stage decisions $Q^{\mathcal{R}^{*}}\left(\boldsymbol{\eta}_{\boldsymbol{\mu}}, \boldsymbol{\eta}_{\boldsymbol{\sigma}^{2}}\right)$. It is defined as

$$
\mathbb{E} \mathcal{R}\left(\boldsymbol{\eta}_{\boldsymbol{\mu}}, \boldsymbol{\eta}_{\boldsymbol{\sigma}^{2}}\right)=\mathbb{E}_{\mathcal{N}_{\mathcal{R}}\left(\boldsymbol{\eta}_{\boldsymbol{\mu}} \boldsymbol{\mu}, \boldsymbol{\eta}_{\boldsymbol{\sigma}^{2}} \sigma^{2}\right)} \mathcal{C}\left(Q^{\mathcal{R}^{*}}\left(\boldsymbol{\eta}_{\boldsymbol{\mu}}, \boldsymbol{\eta}_{\boldsymbol{\sigma}^{2}}\right)\right)
$$


3. The performance bound of using the guessed distribution $\mathcal{N}_{\mathcal{G}}\left(\boldsymbol{\mu}, \boldsymbol{\sigma}^{\mathbf{2}}\right)$ represents the loss in using the wrong guessed distribution, compared to the right distribution $\mathcal{N}_{\mathcal{R}}$. It is defined as

$$
P B_{\mathcal{N}_{\mathcal{G}}, \mathcal{N}_{\mathcal{R}}}\left(\boldsymbol{\eta}_{\boldsymbol{\mu}}, \boldsymbol{\eta}_{\sigma^{2}}\right)=\frac{\mathbb{E} \mathcal{R}\left(\boldsymbol{\eta}_{\boldsymbol{\mu}}, \boldsymbol{\eta}_{\boldsymbol{\sigma}^{2}}\right)}{\mathbb{E} \mathcal{G}\left(\boldsymbol{\eta}_{\boldsymbol{\mu}}, \boldsymbol{\eta}_{\boldsymbol{\sigma}^{2}}\right)}
$$

The performance bounds of the guessed distribution are computed for all combinations of error factors with the proposed electricity-aware heat market-clearing model, as represented in Figures 8 and 9. Note that the variations can be explained by the sampling effect, and the specific 1,000 scenarios selected to computed the average system costs. However, these figures show that the proposed model still achieves more than $95 \%$ of the expectation of the right distribution for errors in the mean and variance of the guessed distributions up to $50 \%$.
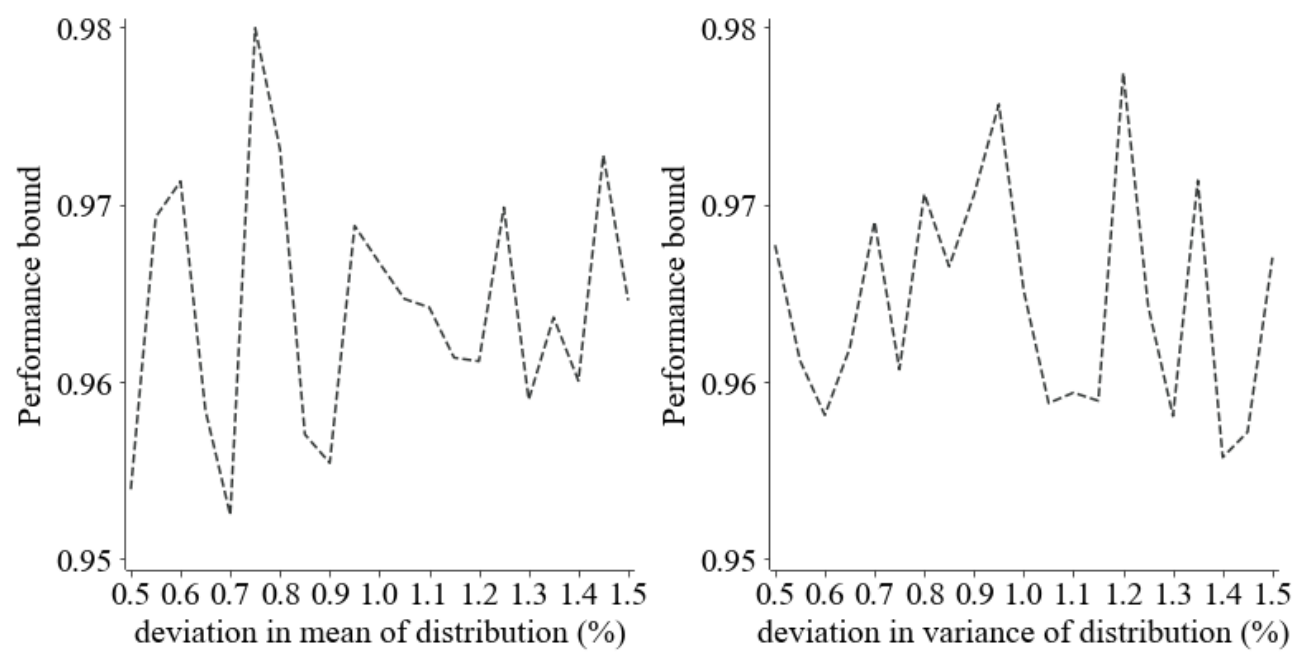

Figure 8: Performance bounds of the guessed distribution with respect to varying error in (a) mean value of electricity load factors $\left(\eta_{\mu^{L} E}\right)$ and (b) variance of electricity load factors $\left.\left(\eta_{\left(\sigma^{L} E\right.}\right)^{2}\right)$.

\section{Conclusions}

This study deals with the coordination of heat and renewable-based power systems via marketbased mechanisms. It uses two reference market models: a traditional sequential market framework and a fully integrated market framework. The heat-driven dispatch in the sequential market framework limits the flexibility of CHPs and HPs, participating in both markets. The fully integrated market, though providing an ideal benchmark for the heat and power dispatch, goes against the current market regulations.

To bridge this gap, this study introduces a novel market framework based on a hierarchical optimization model that endogenously models electricity market clearing as a constraint of the heat market optimization problem. Our analysis prompts two fundamental conclusions. First, there is room for significant improvement in the heat and electricity market coordination, while respecting current market regulations. The proposed electricity-aware market framework provides a trade-off between the traditional sequential market and a fully integrated one, by providing insights to the heat market operator into the impact of heat dispatch on the power system. Second, the proposed 

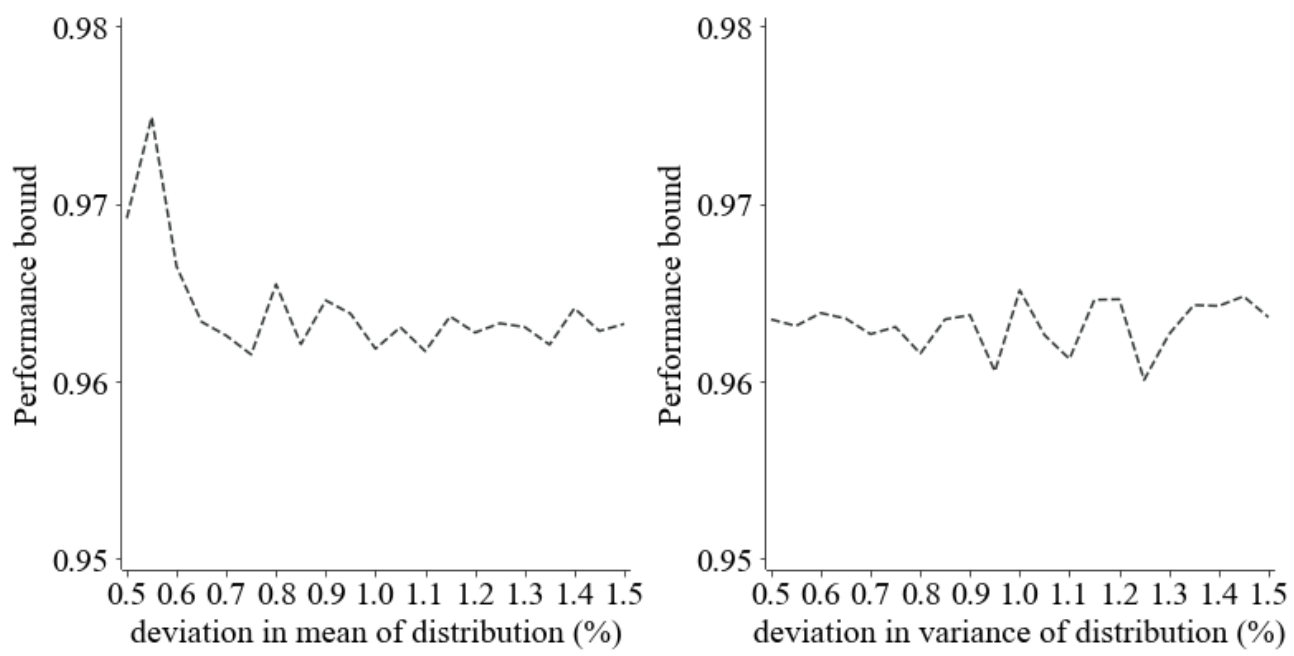

Figure 9: Performance bounds of the guessed distribution with respect to varying error in (a) mean value of electricity supply curve $\left(\eta_{\mu^{\alpha}}\right.$ ) and (b) variance of electricity supply curve $\left(\eta_{\left(\sigma^{\alpha}\right)^{2}}\right)$.

stochastic electricity-aware market framework can be solved in an efficient and computationally tractable manner using an augmented regularized Benders algorithm developed in this study. This allows us to model the sources of uncertainty from the power system using a large number of scenarios without loss of tractability.

This work shows that a coordinated operation of heat and electricity systems can provide flexibility to the overall energy system in order to accommodate more stochastic and non-dispatchable renewable energy sources. This opens up various directions for future research, including proposing novel mathematical models for more accurate representation of different components of the systems, as well as redesigning the markets towards a better coordination between different energy systems and market floors. Firstly, due to the lack of real data, the numerical results should be considered with caution and further analyses should be conducted on real complex energy systems. Secondly, various existing studies in the literature suggest that a more accurate representation of the underlying physics of district heating networks and the techno-economic characteristics of heat producers in a market environment would reveal additional operational flexibility, create financial opportunities for harnessing this flexibility in a market environment, and reduce inefficiencies in the operation of heat and electricity systems (Dai et al., 2018; Mitridati \& Taylor, 2018; Rong \& Lahdelma, 2016; Li et al., 2016b). Accurately accounting for these characteristics raises major computational and pricing issues due to non-convexities in a market environment. Therefore, following the work by Byeon \& Van Hentenryck (2019b), a promising research direction would be to develop an electricity-aware unit commitment model for heat producers and CHPs. Integrating such comprehensive operational models in a market environment requires the development of advanced mathematical models for large-scale non-convex optimization problems based on convex relaxation (Mitridati \& Taylor, 2018; Sanchez et al., 2016) and decomposition methods (Byeon \& Van Hentenryck, 2019a; Li et al., 2016a; Abdollahi et al., 2019; Loukarakis \& Mancarella, 2017).

Moreover, this study focuses on the issue of coordination between energy systems at the dayahead market stage, however it does not account for real-time uncertainty. One way to achieve this would be to readily account for coordinated heat and electricity reserve requirements at the 
day-ahead stage. Warrington et al. (2013) and Darivianakis et al. (2017) introduce policy-based reserve products, defined as affine policies. This approach could provide an appropriate framework to model the flexibility of CHPs, HPs, and consumers at the interface between heat and power systems, and coordinate their response to disturbances from both systems.

Finally, while this study targets market design, other approaches could be investigated to improve the coordination between heat and power systems within the existing market framework. In particular, developing flexible products can provide a soft link between energy systems. Li et al. (2016a); Mitridati \& Taylor (2018); Dai et al. (2018) show that the inter-temporal flexibility of district heating networks, coupled with CHPs and HPs at the interface between heat and power systems, could be modeled as a virtual electricity storage. Hence, a promising direction is to generalize the concept of financial and physical storage rights developed by Taylor (2015) and Muñoz-Álvarez \& Bitar (2017), to allow heat systems to quantify and offer their flexibility to the power system, in the form of a virtual electricity storage.

\section{Appendix: Nomenclature}

\section{Sets and Indexes}

$\mathcal{T}$ Set of time periods in the day-ahead market, i.e., 24 hours of the following day

$\mathcal{X}$ Set of day-ahead scenarios

$\mathcal{I}^{\mathbf{H}}$ Set of heat market participants

$\mathcal{I}^{\text {HO }}$ Set of heat-only units

$\mathcal{I}^{\text {HS }}$ Set of heat storage tanks

$\mathcal{I}^{\mathrm{CHP}}$ Set of CHPs

$\mathcal{I}^{\text {HP }}$ Set of HPs

$\mathcal{I}^{\mathbf{E}}$ Set of thermal power plants and wind power producers

$\Omega^{\text {DA }}$ Set of day-ahead heat dispatch variables

$\Omega_{\nu}^{\mathbf{L L}}$ Set of primal optimization variables of the day-ahead electricity market under scenario $\nu$

$\Xi_{\nu}^{\mathbf{L L}}$ Set of dual optimization variables of the day-ahead electricity market under scenario $\nu$

$\Omega_{\nu}^{\mathbf{R}}$ Set of heat redispatch variables under scenario $\nu$

$\Omega_{\nu}^{\text {Int }}$ Set of optimization variables of integrated heat and electricity market under scenario $\nu$

$\Omega^{\text {UL }}$ Set of optimization variables of the upper-level problem in the electricity-aware heat market clearing

\section{Input Parameters}


$\pi_{\nu}$ Probability of scenario $\nu$

$\bar{Q}_{j}$ Maximum heat output of CHP, or HP, or heat-only unit, or heat storage tank $j$ (MW)

$\rho_{j}^{\mathbf{H}}, \rho_{j}^{\mathbf{E}}$ Heat and electricity fuel efficiency of CHP $j$

$r_{j}$ Heat to electricity ratio of $\mathrm{CHP} j$

$\bar{F}_{j}$ Maximum fuel consumption of CHP $j(\mathrm{MW})$

$\mathbf{C O P}_{j}$ Coefficient of performance of heat pump $j$

$\bar{S}_{j}$ Maximum heat stored in heat storage tank $j(\mathrm{MWh})$

$\underline{S}_{j}$ Minimum heat stored in heat storage tank $j(\mathrm{MWh})$

$S_{j}^{\text {init }}$ Initial heat stored in heat storage tank $j(\mathrm{MWh})$

$\rho_{j}^{-}, \rho_{j}^{+}$Charging and discharging efficiencies of heat storage tank $j$

$l_{j}$ Losses of heat storage tank $j(\mathrm{MWh})$

$L_{t \nu}^{\mathbf{E}} \quad$ Electricity load scenario in the day-ahead market at time period $t$ under scenario $\nu(\mathrm{MW})$

$L_{t}^{\mathbf{H}}$ Heat load in the day-ahead market at time period $t(\mathrm{MW})$

$\bar{P}_{j t \nu}$ Maximum power output of conventional generator or wind power producer $j$ at time period $t$ under scenario $\nu(\mathrm{MW})$

$\underline{\alpha}$ Minimum price offer in the day-ahead electricity market, i.e., $-€ 500 / \mathrm{MWh}$ as in the Nordpool electricity market

$\bar{\alpha}$ Maximum price offer in the day-ahead electricity market, i.e., $€ 3000 /$ MWh as in the Nordpool electricity market

$\alpha_{j}$ Marginal cost parameter of CHP or heat-only unit $j(€ / \mathrm{MWh})$

$\tilde{\alpha}_{j t \nu}^{\mathbf{E}}$ Marginal price offer of conventional generator or wind power producer $j$ in the day-ahead electricity market at time period $t$ under scenario $\nu(€ / \mathrm{MWh})$

$\alpha_{j}^{\mathbf{E}}$ Electricity marginal cost of $\mathrm{CHP} j(€ / \mathrm{MWh})$

$\alpha_{j}^{\uparrow}, \alpha_{j}^{\downarrow} \mathrm{Up}$ and down redispatch costs of CHP, or HP, or heat-only unit $j$ (€/MWh)

$\hat{\lambda}_{t}^{\mathbf{E}}$ Forecasted electricity price at time period $t(€ / \mathrm{MWh})$

$\mu_{t}^{L^{E}}$ Mean of distribution of electricity load at time period $t$

$\left(\sigma_{t}^{L^{E}}\right)^{2}$ Variance of distribution of electricity load at time period $t$

$\mu_{j t}^{\alpha^{E}}$ Mean of distribution of electricity supply curve at time period $t$ for market participant $j$

$\left(\sigma_{j t}^{\alpha^{E}}\right)^{2}$ Variance of electricity supply curve at time period $t$ for market participant $j$ 
$\eta_{\mu_{t}^{L} E}$ Error factor of mean of distribution of electricity load at time period $t$

$\eta_{\left(\sigma_{t}^{L}\right)^{2}}$ Error factor of variance of distribution of electricity load at time period $t$

$\eta_{\mu_{j t}^{\alpha E}}$ Error factor of mean of distribution of electricity supply curve at time period $t$ for market participant $j$

$\eta_{\left(\sigma_{j t}^{\alpha E}\right)^{2}}$ Error factor of variance of electricity supply curve at time period $t$ for market participant $j$

\section{Decision variables}

$Q_{j t}$ Day-ahead heat dispatch of CHP, or HP, or heat-only unit $j$ at time period $t$ (MWh)

$Q_{j t}^{-}, Q_{j t}^{+}$Charging and discharging of heat storage tank $j$ in the day-ahead market at time period $t$ (MWh)

$S_{j t}$ Heat stored in heat storage tank $j$ in the day-ahead market at time period $t(\mathrm{MWh})$

$Q_{j t \nu}^{\uparrow}, Q_{j t \nu}^{\downarrow}$ Upward and downward heat production adjustment of CHP or heat-only unit $j$ at time period $t$ under scenario $\nu$ (MWh)

$Q_{j t \nu}$ Heat production of CHP or heat-only unit $j$ after redispatch at time period $t$ under scenario $\nu(\mathrm{MWh})$

$Q_{j t \nu}^{+}, Q_{j t \nu}^{-}$Charging and discharging of heat storage tank $j$ after redispatch at time period $t$ under scenario $\nu(\mathrm{MWh})$

$S_{j t \nu}$ Heat stored in heat storage tank $j$ after redispatch at time period $t$ under scenario $\nu(\mathrm{MWh})$

$P_{j t \nu}$ Day-ahead electricity dispatch of conventional generator, or wind power producer, or CHP $j$ at time period $t$ under scenario $\nu(\mathrm{MWh})$

$P_{j t \nu}^{0}$ Electricity production of CHP $j$ below $\underline{P}_{j}\left(Q_{j t}\right)$ at time period $t$ under scenario $\nu(\mathrm{MWh})$

$P_{j t \nu}^{+}$Electricity production of CHP $j$ over $\underline{P}_{j}\left(Q_{j t}\right)$ at time period $t$ under scenario $\nu(\mathrm{MWh})$

$L_{j t \nu}^{\mathbf{H P}}$ Electricity consumption of HP $j$ at time period $t$ under scenario $\nu$ (MWh)

$\underline{\mu}_{j t \nu}, \bar{\mu}_{j t \nu}, \underline{\mu}_{j t \nu}^{0}, \bar{\mu}_{j t \nu}^{0}$ Dual variables of the lower-level problems

$\lambda_{t \nu}^{\mathbf{E}}$ Day-ahead electricity price at time period $t$ under scenario $\nu$

$\lambda_{t \nu}^{\mathbf{H}}$ Day-ahead heat price at time period $t$ under scenario $\nu$

\section{Functions}


$\Gamma_{j}($.$) Total production cost of CHP j$, depending on electricity prices, heat and electricity production $(€)$

$\Gamma_{j}^{\mathbf{H}}($.$) Expected heat cost of CHP or HP j$, depending on electricity prices, heat and electricity production $(€)$

$\alpha_{j}^{\mathbf{H}}($.$) Expected heat marginal cost of CHP or HP j$, depending on electricity prices $(€ / \mathrm{MWh})$

$\bar{P}_{j}($.$) Maximum power output of CHP j$, depending on heat output (MW)

$\underline{P}_{j}($.$) Minimum power output of CHP j$, depending on heat output (MW)

$\underline{L}_{j}^{\mathbf{H P}}($.$) Minimum power consumption of HP j$, depending on heat output (MW)

$\mathcal{N}(.,$.$) Normal distribution, characterized by its mean and variance$

\section{Benders algorithm indexes, variables and parameters}

$\theta$ Iterations of Benders algorithm

$\Omega^{\mathrm{MP}}$ Set of optimization variables of master problem

$\Omega_{\nu}^{\text {SUB }}$ Set of optimization variables of subproblem $\mathrm{SUB}_{\nu}$ under scenario $\nu$

$\epsilon$ A non-negative tolerance parameter

$\eta_{j t \nu}^{Q,(\theta)}$ Sensitivity of subproblem $\mathrm{SUB}_{\nu}$ with respect to complicating variable $Q_{j t}$ at iteration $\theta$

$\eta_{j t \nu}^{S,(\theta)}$ Sensitivity of subproblem $\mathrm{SUB}_{\nu}$ with respect to complicating variable $S_{j t}$ at iteration $\theta$

$\eta_{j t \nu}^{Q^{+},(\theta)}$ Sensitivity of subproblem $\mathrm{SUB}_{\nu}$ with respect to complicating variable $Q_{j t}^{+}$at iteration $\theta$

$\eta_{j t \nu}^{Q^{-},(\theta)}$ Sensitivity of subproblem $\mathrm{SUB}_{\nu}$ with respect to complicating variable $Q_{j t}^{-}$at iteration $\theta$

$z_{\nu}^{\mathrm{SUB},(\theta)}$ Objective value of $\mathrm{SUB}_{\nu}$ at iteration $\theta$

$z^{\mathrm{MP},(\theta)}$ Objective value of master problem at iteration $\theta$

$\mathbf{U B}^{(\theta)}$ Value of upper bound at iteration $\theta$

$\mathbf{L B}^{(\theta)}$ Value of lower bound at iteration $\theta$

$X^{(\text {ref })}$ Reference point

$\tau^{\text {(ref) }}$ Penalization parameter

$m$ Regularization parameter $(0<m<1)$

$X$ Vector of complicating variables

$\beta$ An auxiliary variable in the master problem 


\section{References}

Abdollahi, E., Wang, H., \& Lahdelma, R. (2019). Parametric optimization of long-term multi-area heat and power production with power storage. Applied Energy, 235, 802-812.

Ackermann, T. (2005). Wind Power in Power Systems. John Wiley \& Sons.

Bach, B., Werling, J., Ommen, T., Münster, M., Morales, J. M., \& Elmegaard, B. (2016). Integration of large-scale heat pumps in the district heating systems of greater copenhagen. Energy, 10\%, 321-334.

Benders, J. F. (1962). Partitioning procedures for solving mixed-variables programming problems. Numerische Mathematik, 4, 238-252.

Bertsekas, D. P., \& Sandell, N. R. (1982). Estimates of the duality gap for large-scale separable nonconvex optimization problems. In 21st IEEE Conference on Decision and Control (pp. 782-785).

Bonnans, J.-F., Gilbert, J. C., Lemaréchal, C., \& Sagastizábal, C. A. (2006). Numerical Optimization: Theoretical and Practical Aspects. Springer Science \& Business Media.

Bukhsh, W. (2015). Data for stochastic multiperiod optimal power flow problem. URL: http://sites.google.com/ site/datasmopf/.

Bukhsh, W. A., Zhang, C., \& Pinson, P. (2016). An integrated multiperiod opf model with demand response and renewable generation uncertainty. IEEE Transactions on Smart Grid, 7, 1495-1503.

Byeon, G., \& Van Hentenryck, P. (2019a). Benders decomposition for a class of mathematical programs with constraints on dual variables. In arXiv preprint arXiv:1902.04375.

Byeon, G., \& Van Hentenryck, P. (2019b). Unit commitment with gas network awareness. IEEE Transactions on Power Systems, (pp. 1-1). To be published.

Castillo, E., Mínguez, R., Conejo, A., \& Garcia-Bertrand, R. (2006). Decomposition Techniques in Mathematical Programming. Springer.

Chen, R., Paschalidis, I. C., Caramanis, M. C., \& Andrianesis, P. (2019). Learning from past bids to participate strategically in day-ahead electricity markets. IEEE Transactions on Smart Grid, .

Chen, X., Kang, C., O’Malley, M., Xia, Q., Bai, J., Liu, C., Sun, R., Wang, W., \& Li, H. (2015). Increasing the flexibility of combined heat and power for wind power integration in China: Modeling and implications. IEEE Transactions on Power Systems, 30, 1848-1857.

Chu, S., \& Majumdar, A. (2012). Opportunities and challenges for a sustainable energy future. Nature, 488, $294-303$.

Cruz, J. (1978). Leader-follower strategies for multilevel systems. IEEE Transactions on Automatic Control, 23, 244-255.

Dai, Y., Chen, L., Min, Y., Mancarella, P., Chen, Q., Hao, J., Hu, K., \& Xu, F. (2018). A general model for thermal energy storage in combined heat and power dispatch considering heat transfer constraints. IEEE Transactions on Sustainable Energy, 9, 1518-1528.

Darivianakis, G., Georghiou, A., Eichler, A., Smith, R. S., \& Lygeros, J. (2017). Scalability through decentralization: A robust control approach for the energy management of a building community. IFAC-PapersOnLine, 50, 1431414319.

Gabriel, S. A., Conejo, A. J., Fuller, J. D., Hobbs, B. F., \& Ruiz, C. (2012). Complementarity Modeling in Energy Markets. Springer.

Gabriel, S. A., Zhuang, J., \& Egging, R. (2009). Solving stochastic complementarity problems in energy market modeling using scenario reduction. European Journal of Operational Research, 197, 1028-1040.

Geoffrion, A. M. (1972). Generalized Benders decomposition. Journal of Optimization Theory and Applications, 10, $237-260$.

Kazempour, J., \& Conejo, A. J. (2012). Strategic generation investment under uncertainty via Benders decomposition. IEEE Transactions on Power Systems, 27, 424-432.

Lahdelma, R., \& Hakonen, H. (2003). An efficient linear programming algorithm for combined heat and power production. European Journal of Operational Research, 148, 141-151.

Li, Z., Wu, W., Shahidehpour, M., Wang, J., \& Zhang, B. (2016a). Combined heat and power dispatch considering pipeline energy storage of district heating network. IEEE Transactions on Sustainable Energy, 7, 12-22.

Li, Z., Wu, W., Wang, J., Zhang, B., \& Zheng, T. (2016b). Transmission-constrained unit commitment considering combined electricity and district heating networks. IEEE Transactions on Sustainable Energy, 7, 480-492.

Loukarakis, E., \& Mancarella, P. (2017). A sequential programming method for multi-energy districts optimal power flow. In 2017 IEEE Manchester PowerTech (pp. 1-6). IEEE.

Lund, H. (2005). Large-scale integration of wind power into different energy systems. Energy, 30, 2402-2412.

Lund, H., Möller, B., Mathiesen, B. V., \& Dyrelund, A. (2010). The role of district heating in future renewable energy systems. Energy, 35, 1381-1390. 
Madsen, H. (2015). Time series analysis. course notes. URL: http://www.imm.dtu.dk/ hmad/time.series. analysis/assignments/index.html.

Maggioni, F., Cagnolari, M., \& Bertazzi, L. (2019). The value of the right distribution in stochastic programming with application to a newsvendor problem. Computational Management Science, (pp. 1-20).

Meibom, P., Hilger, K. B., Madsen, H., \& Vinther, D. (2013). Energy comes together in Denmark: The key to a future fossil-free Danish power system. IEEE Power Energy Magazine, 11, 46-55.

Meibom, P., Kiviluoma, J., Barth, R., Brand, H., Weber, C., \& Larsen, H. V. (2007). Value of electric heat boilers and heat pumps for wind power integration. Wind Energy, 10, 321-337.

Mitridati, L., Kazempour, J., \& Pinson, P. (2019). Heat and electricity market coordination: A scalable complementarity approach - python code and online appendix. URL: https://doi.org/10.5281/zenodo.2769456.

Mitridati, L., \& Pinson, P. (2016). Optimal coupling of heat and electricity systems: A stochastic hierarchical approach. In 2016 International Conference on Probabilistic Methods Applied to Power Syst. (PMAPS). IEEE.

Mitridati, L., \& Pinson, P. (2018). A Bayesian inference approach to unveil supply curves in electricity markets. IEEE Transactions on Power Systems, 33, 2610-2620.

Mitridati, L., \& Taylor, J. A. (2018). Power systems flexibility from district heating networks. In 2018 Power Systems Computation Conference (PSCC) (pp. 1-7). IEEE.

Morales, J. M., Conejo, A. J., Madsen, H., Pinson, P., \& Zugno, M. (2013). Integrating Renewables in Electricity Markets. Springer Science \& Business Media.

Morales, J. M., Pineda, S., Conejo, A. J., \& Carrion, M. (2009). Scenario reduction for futures market trading in electricity markets. IEEE Transactions on Power Systems, 24, 878-888.

Muñoz-Álvarez, D., \& Bitar, E. (2017). Financial storage rights in electric power networks. Journal of Regulatory Economics, 52, 1-23.

Nasri, A., Kazempour, J., Conejo, A. J., \& Ghandhari, M. (2016). Network-constrained AC unit commitment under uncertainty: a Benders' decomposition approach. IEEE Transactions on Power Systems, 31, 412-422.

Nordpool (2018). Market data - consumption. URL: https://www.nordpoolgroup.com/Market-data1/ Power-system-data/Consumption1/Consumption/ALL/Hourly1/?view=table.

Nordpool (2019). Elspot - single hourly order. URL: https://www.nordpoolgroup.com/trading/ Day-ahead-trading/Order-types/Hourly-bid/.

Ordoudis, C., Delikaraoglou, S., Pinson, P., \& Kazempour, J. (2017). Exploiting flexibility in coupled electricity and natural gas markets: A price-based approach. In 2017 IEEE Manchester PowerTech (pp. 1-6). IEEE.

Ordoudis, C., Pinson, P., Morales, J. M., \& Zugno, M. (2016). An updated version of the IEEE RTS 24-bus system for electricity market and power system operation studies - DTU working paper (available online). URL: http://orbit.dtu.dk/files/120568114/An.

Pantuso, G., Fagerholt, K., \& Wallace, S. W. (2015). Which uncertainty is important in multistage stochastic programmes? A case from maritime transportation. IMA Journal of Management Mathematics, 28, 5-17.

Pinson, P. (2013). Wind energy: Forecasting challenges for its operational management. Statistical Science, 28, $564-585$.

Pinson, P., Mitridati, L., Ordoudis, C., \& Østergaard, J. (2017). Towards fully renewable energy systems: Experience and trends in Denmark. CSEE Journal of Power and Energy Systems, 3, 26-35.

Rahmaniani, R., Crainic, T. G., Gendreau, M., \& Rei, W. (2017). The Benders decomposition algorithm: A literature review. European Journal of Operational Research, 259, 801-817.

Rey, P. A., \& Sagastizábal, C. (2002). Dynamical adjustment of the prox-parameter in bundle methods. Optimization, $51,423-447$.

Rong, A., \& Lahdelma, R. (2016). Role of polygeneration in sustainable energy system development challenges and opportunities from optimization viewpoints. Renewable and Sustainable Energy Reviews, 53, 363-372.

Ruszczynski, A. (1986). A regularized decomposition method for minimizing a sum of polyhedral functions. Mathematical Programming, 35, 309-333.

Ruszczynski, A., \& Swietanowski, A. (1997). Accelerating the regularized decomposition method for two stage stochastic linear problems. European Journal of Operational Research, 101, 328-342.

Sanchez, C. B., Bent, R., Backhaus, S., Blumsack, S., Hijazi, H., \& Van Hentenryck, P. (2016). Convex optimization for joint expansion planning of natural gas and power systems. In 2016 49th Hawaii International Conference on System Sciences (HICSS) (pp. 2536-2545). IEEE.

Simaan, M., \& Cruz, J. B. (1973a). Additional aspects of the stackelberg strategy in nonzero-sum games. Journal of Optimization Theory and Applications, 11, 613-626.

Simaan, M., \& Cruz, J. B. (1973b). On the Stackelberg strategy in nonzero-sum games. Journal of Optimization Theory and Applications, 11, 533-555. 
von Stackelberg, H. (1934). Marktjorm und Gleichgewicht. Springer.

Taylor, J. A. (2015). Financial storage rights. IEEE Transactions on Power Systems, 30, 997-1005.

Taylor, J. A., Dhople, S. V., \& Callaway, D. S. (2016). Power systems without fuel. Renewable and Sustainable Energy Reviews, 57, 1322-1336.

Ummels, B. C., Gibescu, M., Pelgrum, E., Kling, W. L., \& Brand, A. J. (2007). Impacts of wind power on thermal generation unit commitment and dispatch. IEEE Transactions on Energy Conversion, 22, 44-51.

Varmelast (2018). Heating plans. URL: http://varmelast.dk/en/heat-plans/heating-plans.

Virasjoki, V., Siddiqui, A. S., Zakeri, B., \& Salo, A. (2018). Market power with combined heat and power production in the Nordic energy system. IEEE Transactions on Power Systems, 33, 5263-5275.

Wang, J., Zong, Y., You, S., \& Træholt, C. (2017). A review of Danish integrated multi-energy system flexibility options for high wind power penetration. Clean Energy, 1, 23-35.

Warrington, J., Goulart, P., Mariéthoz, S., \& Morari, M. (2013). Policy-based reserves for power systems. IEEE Transactions on Power Systems, 28, 4427-4437.

Weber, C. (2006). Uncertainty in the electric power industry: methods and models for decision support volume 77. Springer Science \& Business Media.

Wu, X., \& Conejo, A. J. (2017). An efficient tri-level optimization model for electric grid defense planning. IEEE Transactions on Power Systems, 32, 2984-2994.

Zugno, M., Morales, J. M., \& Madsen, H. (2016). Commitment and dispatch of heat and power units via affinely adjustable robust optimization. Computers $\&$ Operations Research, 75, 191-201. 